\title{
基于石墨烯的电磁波损耗材料研究进展
}

\author{
康 越 ${ }^{1,2}$, 原 博 $^{3}$, 马 天 $^{2}$, 楚增勇 ${ }^{4}$, 张政军 ${ }^{1}$
}

(1. 清华大学 材料学院, 北京 $100084 ; 2$. 军事科学院 军需工程技术研究所, 北京 $100010 ; 3$. 军事科学院 防化研 究院, 北京 100010; 4. 国防科技大学 文理学院, 长沙 410073)

摘 要: 随着军事装备隐身伪装需求的迅速增长, 石墨烯基电磁波损耗材料成为科学研究的热点。本文总结了石墨 烯基电磁波损耗材料的研究进展, 详细介绍了石墨烯与其它碳系材料、金属纳米材料、铁氧体材料、导电高聚物材 料及非金属材料复合而成的二元、三元及以上多元石墨烯基电磁波损耗材料。简要阐述了电磁波损耗机理, 着重讨 论了石墨烯基电磁波损耗材料的微观结构、组分数量及不同损耗机制对吸波性能的影响，展望了石墨烯基电磁波损 耗材料的研究重点和发展方向。

关 键 词: 石墨烯; 石墨烯复合吸波材料; 吸波性能; 综述

中图分类号: TB34 文献标识码: A

\section{Development of Microwave Absorbing Materials Based on Graphene}

\author{
KANG Yue ${ }^{1,2}$, YUAN Bo $^{3}$, MA Tian $^{2}$, CHU Zeng-Yong ${ }^{4}$, ZHANG Zheng-Jun $^{1}$
}

\begin{abstract}
(1. School of Materials Science and Engineering, Tsinghua University, Beijing 100084, China; 2. Quartermaster Equipment Research Institute, Academy of Military Sciences PLA China, Beijing 100010, China; 3. Chemical Defense Institute of China, Academy of Military Sciences PLA China, Beijing 100010, China; 4. College of Liberal Arts and Sciences, National University of Defense Technology, Changsha 410073, China)
\end{abstract}

\begin{abstract}
Nowadays, electromagnetic interference receives great attention for wireless communication and charging, electronic device, and modern weapons. Materials and the various novelty structures are required and designed to absorb the emitted electromagnetic wave energy and to minimize reflection of the electromagnetic wave in certain direction. Development of graphene-based electromagnetic absorption materials are reviewed, consisting of composition materials with other carbon materials, nano-metal materials, ferrite materials, polymer materials, and non-metallic materials. The electromagnetic absorption mechanisms is briefly described and the influences of microstructure, fraction and loss mechanism on the absorption properties are discussed. The future of graphene-based electromagnetic absorption materials is also prospected.
\end{abstract}

Key words: graphene; electromagnetic wave absorbing materials; absorption property; review

石墨烯作为二维单层碳原子材料, 是构成碳材 料家族的最基本单元, 将石墨烯堆叠, 可得到六方 石墨; 将其卷曲, 又可得到零维富勒烯或一维碳纳 米管。科学工作者对石墨烯及其衍生物的研究最早 可追溯到 1840 年, 但直到 Geim 和 Novoselov 首次 制备石墨烯以来, 石墨烯才受到全世界科学家的广
泛关注 ${ }^{[1-3]}$ 。

\section{1 石墨烯及其衍生物的基本性质}

石墨烯轻质层薄, 理论厚度只有 $0.35 \mathrm{~nm}^{[4]}$ 。表 面无含氧基团, 呈化学反应惰性, 耐高温且不易氧

收稿日期：2018-04-25; 收到修改稿日期：2018-07-04

基金项目：国家自然科学基金青年基金项目(11404397) National Natural Science Foundation of China (11404397)

作者简介：康 越(1989-), 男, 工程师, 博士研究生. E-mail: goodluckky@163.com

通讯作者: 楚增勇, 研究员. E-mail: chuzy@nudt.edu.cn; 张政军, 教授. E-mail: zjzhang@tsinghua.edu.cn 
化。石墨烯具备优异的电学特性, 载流子迁移率超 过商用硅片 10 倍。单层石墨烯是零带隙导体, 随着 石墨烯层数的增加, 由多层石墨烯叠加形成的材料 带隙约为 $0.04 \mathrm{eV}$, 具备一定半导体特征 ${ }^{[5-8]}$ 。

目前可基本实现低成本量产化石墨烯，常用的 合成方法有 ${ }^{[9-10]}$ : 机械剥离法、加热 $\mathrm{SiC}$ 法、外延 生长法、化学气相沉积法、氧化石墨烯(rGO)还原法、 有机合成法、碳纳米管切割法、低温膨化法和 PMMA 固相生成法等。其中, 氧化石墨烯还原法因 目标产物一一还原石墨烯 $(\mathrm{rGO})$ 量大、效率高而被广 泛采用 ${ }^{[11-14]}$ 。

氧化石墨烯是石墨烯最重要的衍生物, 它保留 了 $2 \mathrm{D}$ 的碳骨架层, 并在边缘连接有羟基 $(-\mathrm{OH})$ 、环 氧基 $(\mathrm{C}-\mathrm{O}-\mathrm{C})$ 、羰基 $(-\mathrm{C}=\mathrm{O})$ 和羧基 $(-\mathrm{COOH})$ 等含氧 官能团 ${ }^{[15]}$ 。氧化石墨烯较石墨烯而言在水溶液或有 机溶液中有非常好的分散性, 且易与其它物质发生 化学反应 ${ }^{[16]}$, 通过与纳米金属粒子、铁氧体或导电 聚合物等发生还原反应或引入缺陷, 可改变带隙结 构, 进而提高电子、离子、分子或界面极化强度, 增 强介电损耗或磁损耗, 从而实现材料对不同频率电 磁波损耗。

\section{2 电磁波损耗材料吸波机理}

微波吸收材料是指能有效接收入射电磁波, 并 使其转化成热能消耗或利用相干相消, 从而使目标 回波强度显著减弱的一类电磁功能材料。吸波材料 性能良好必须具备两个条件: (1)减少电磁波在材料 表面的直接反射, 增加透射强度, 这就需要在设计 材料时充分考虑电磁阻抗匹配特性; (2)电磁波一旦 进入材料内部, 要实现最大限度衰减和损耗, 就需 要考虑材料的损耗特性。根据损耗机制, 吸波材料 可分为介电损耗型和磁损耗型, 介电损耗型吸波材 料主要通过与电场的相互作用而实现电磁波损耗, 吸收率取决于电导损耗、介电弛豫损耗及谐振损耗; 而磁损耗型吸波材料对电磁波的衰减主要来自于畴 壁共振和自然共振、磁滞损耗和后效损耗等磁极化 机制。

目前，常用的微波损耗材料有铁氧体、导电炭 黑、石墨和碳纤维及碳化硅纤维等 ${ }^{[17-18]}$, 但这些材 料存在密度较大、易氧化等固有缺点。故新型微波 吸收材料总是最大限度地追求 “轻、薄、宽、强” 特性。石墨烯的出现最大程度上满足了新型吸波材 料的发展要求, 它轻质、层薄, 电磁波在石墨烯堆 叠、自组装后经层间多次折/反射与表面起伏区/褶
皱区散射协同耗散增大消耗，从而提高纯石墨烯的 吸波性能。同时利用氧化石墨烯优异的反应还原性, 与不同损耗机制(介电型损耗和磁损耗)材料进行多 元复合, 实现对电磁波强损耗的发展要求。

本文针对不同类型石墨烯复合吸波材料的合成 方法和结构性能进行综述, 并对其发展方向和应用 前景进行展望。

\section{3 石墨烯电磁波损耗材料研究进展}

理想的准一维碳纳米管可以看作是由片层状石 墨烯卷曲而来, 科学家对碳纳米管的微波损耗特性 研究要早于石墨烯材料, 研究表明: 由于碳纳米管 电容率较高, 磁导率较低, 故在微波频率下, 较高 的磁导率实部与虚部以及与之相适应的电容率是构 成宽频带吸波材料的基础。而碳纳米管的微波磁导 率相对较低, 限制了其作为微波吸收材料性能的进 一步提高, 因此碳纳米管的本征微波吸收性能较 弱。对碳纳米管进行氟化活化处理可获得最大反射 率为 $-69 \mathrm{~dB}$ 的氟化碳纳米管吸波材料 ${ }^{[19]}$, 且通过调 控碳纳米管长径比、形貌结构或复合金属粒子、铁 氧体、导电高聚物也可提升碳纳米管复合材料对电 磁波的损耗 ${ }^{[20-21]}$ 。所以, 功能化碳纳米管是获得强 吸收、宽频带的碳纳米管微波损耗材料的热点研究 方向。

如图 1 所示, 石墨烯基电磁波损耗材料研究相 对较晚 ${ }^{[22]}$, 经历了由二维结构到三维结构, 二元复 合到多元复合的发展历程。通过调控微观结构和产 物组分, 逐步提升复合材料阻抗匹配性, 对电磁波 实现最大损耗。

\section{1 二维结构石墨烯电磁波损耗材料}

\subsection{1 纯石墨烯材料}

碳系吸波材料主要包括石墨、炭黑、碳纤维、 碳纳米管和石墨烯等, 其中炭黑应用最广, 但作为 吸收剂与石墨相似都有高温抗氧化性差的缺点。碳 纤维和碳纳米管是近年来研究较多的吸波碳材料, 两者兼具结构与功能一体化等诸多优点, 但也存在 表面化学反应惰性, 制备工艺难度大及掺杂改性后 力学性能与吸波性能此消彼长的难题。

与传统的碳材料相比, 石墨烯不仅密度更低、 比表面积更大，而且具有更高的电导率和更好的热 稳定性, 使其有可能取代传统碳材料而成为新型电 磁波吸收材料 ${ }^{[23-29]}$ 。图 2 展示了 $\mathrm{rGO}$ 的典型介电常 数及其反射率曲线, 与其它碳材料类似, 单纯石墨 烯主要以介电损耗为主, 优异的导电性使石墨烯阻抗 


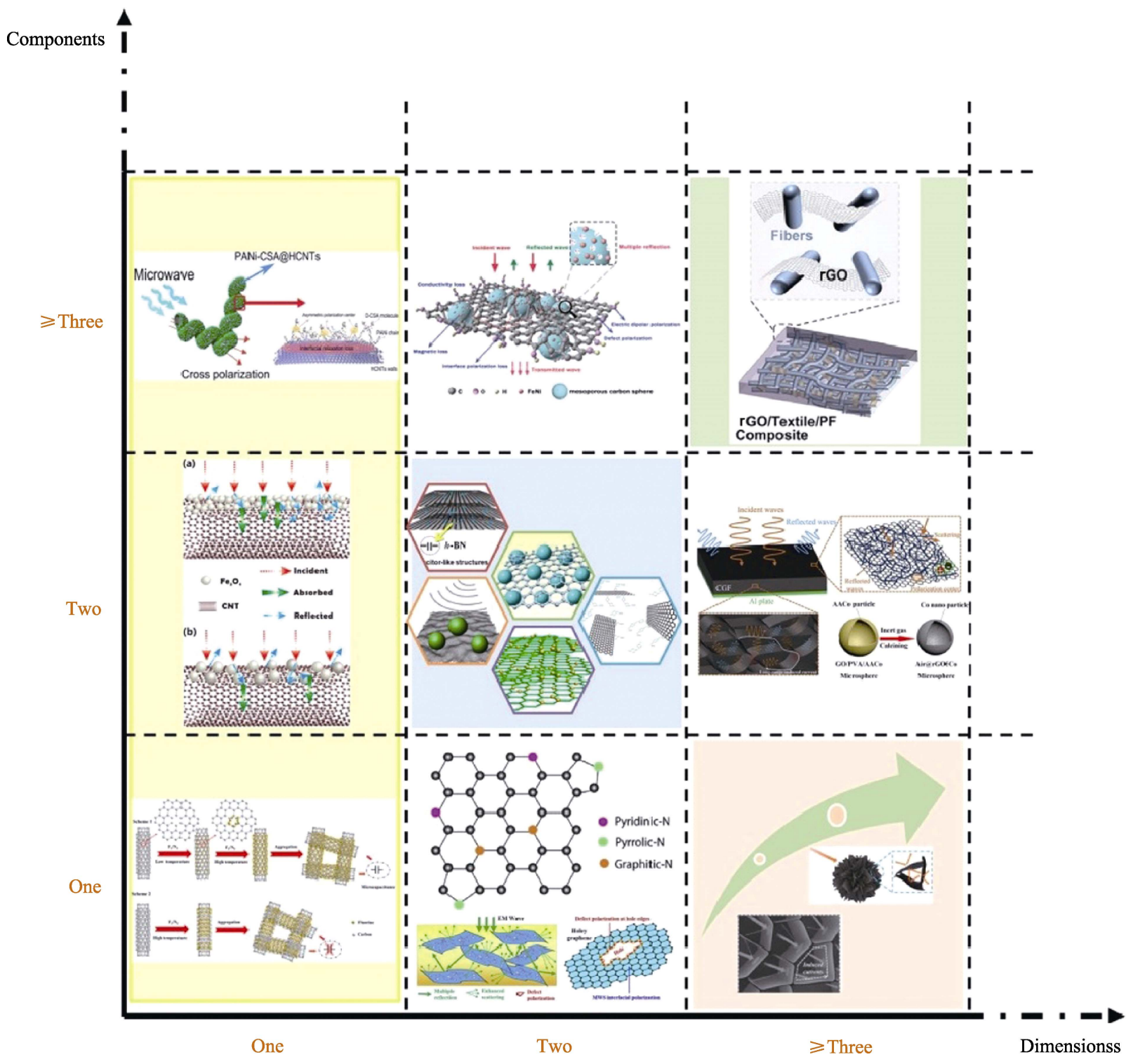

图 1 不同类型和结构的石墨烯基电磁波损耗材料示意图

Fig. 1 Schematic of electromagnetic loss materials based on different types and structures of grapheme

匹配性下降，对电磁波形成反射，吸波性能欠佳 ${ }^{[30]}$ 。 研究表明, 增大比表面积、引入缺陷或改变结构 ${ }^{[31]}$ 可有效提高纯石墨烯材料阻抗匹配性，增强电子从 激发态向费米能级态的跃迁, 增加缺陷处极化驰豫/ 偶极子驰豫，从而提高其吸波性能 ${ }^{[30-34]}$ 。

如图 3 所示, 用导电聚合物替代石蜡作为基体与 石墨烯复合，可增强复合材料电磁波损耗能力 ${ }^{[35-39]}$ 。 这是由于导电聚合物链结构上的偶极子跃迁使其具 有导电性, 电导率的大小取决于导电高分子的分子 链长度及分子结构对偶极子的约束能力, 不同电导 率的复合材料呈现不同的吸波性能。

\subsection{2 复合/掺杂改性石墨烯材料}

\subsubsection{1 石墨烯基二元复合材料}

(1) 石墨烯/纳米碳复合材料

碳纤维和石墨烯组合形成 1D-2D-1D 异质结构
复合材料, 吸波性能较纯石墨烯有较大提升, 最大 反射损耗值为 $-16 \mathrm{~dB}^{[33]}$ 。而如果将石墨烯引入碳纤维 织物内, 所得复合材料最大反射损耗达 $-27.78 \mathrm{~dB}^{[40]}$ 。 不同电导率的碳纳米微球与石墨烯复合可制备吸波 频率可调的复合材料。研究发现层厚为 $1.5 \mathrm{~mm}$ 时, 复合材料的最大反射损耗为 $-28.1 \mathrm{~dB}^{[32]}$ 。

碳纳米管的中空结构增加了电磁波的耗散途径, 增强了界面极化效应，但碳纳米管作为吸波剂与基 体材料复合时易团聚，从而减弱其对电磁波的耗 散。引入石墨烯并以共价 $\mathrm{C}-\mathrm{C}$ 键结合可以改善碳纳 米管分散性，降低表面接触电阻。石墨烯/碳纳米管 多层结构增强复合材料极化效应, 增加介电损耗途 径, 有效提高电磁波损耗能力。结构和形态差异导 致碳纳米管/石墨烯复合材料吸波性能有所不同。煤 焦油沥青基或冻结石油沥青基碳材料包覆的碳纳米 


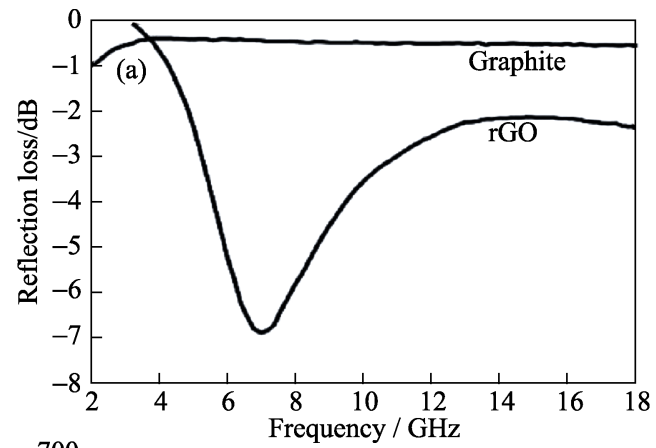

(b)
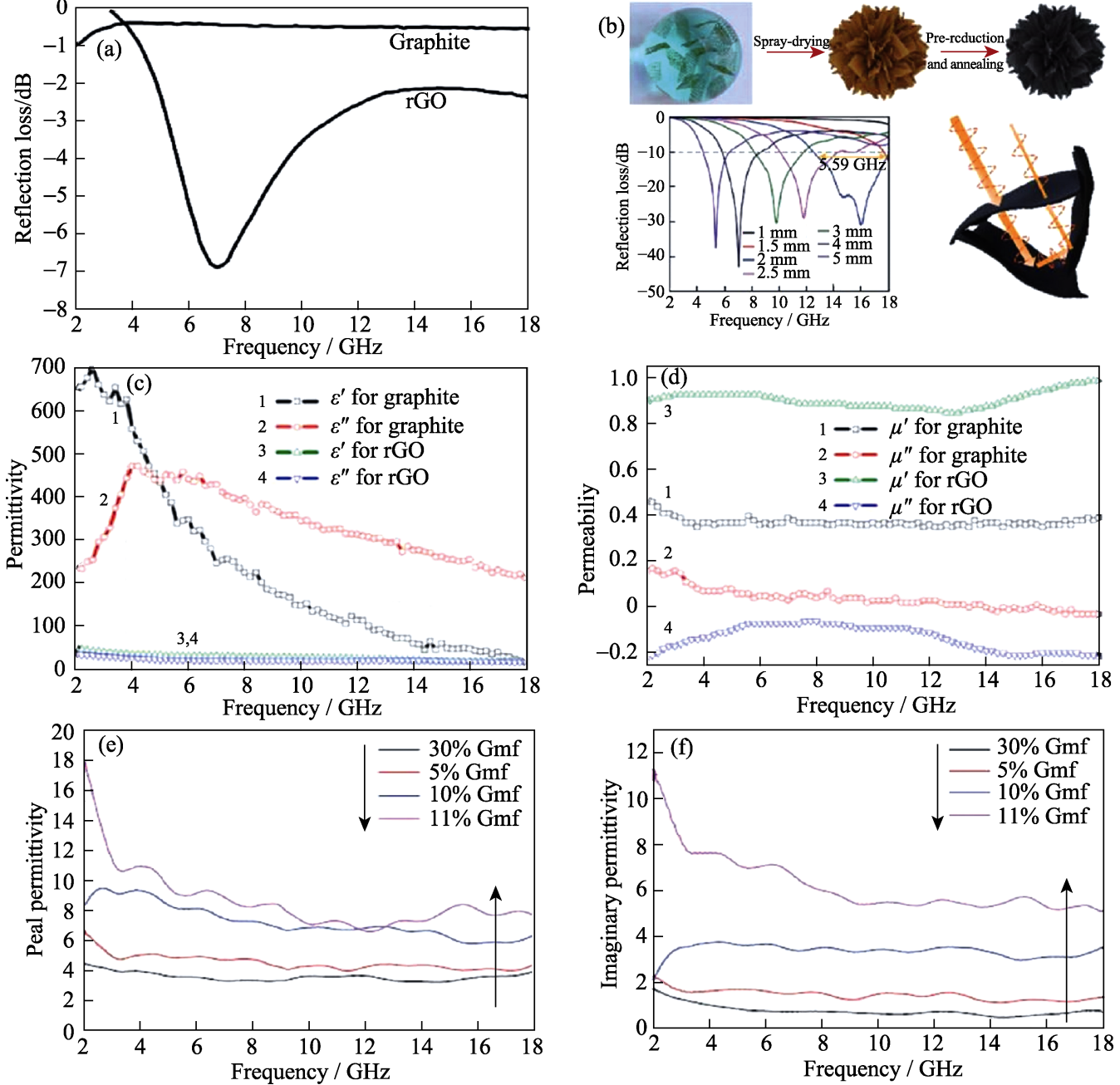

图 $2 \mathrm{rGO}$ 的介电常数与反射率曲线

Fig. 2 Complex permittivity and reflection loss curves of rGO

(a) Reflection loss curves of $\mathrm{rGO}^{[30]}$; (b) Schematic illustration of MA mechanism of $\mathrm{Gmfs}^{[31]}$; (c-d) Frequency dependence of relative complex permittivity real part $\left(\varepsilon^{\prime}\right)(\mathrm{c})$ and imaginary part $\left(\varepsilon^{\prime \prime}\right)(\mathrm{d})$ of graphite and $\mathrm{rGO}^{[30]}$; (e-f) Real parts (e) and imaginary parts

(f) of permittivity of Gmfs/paraffin composites with different filler contents ${ }^{[31]}$
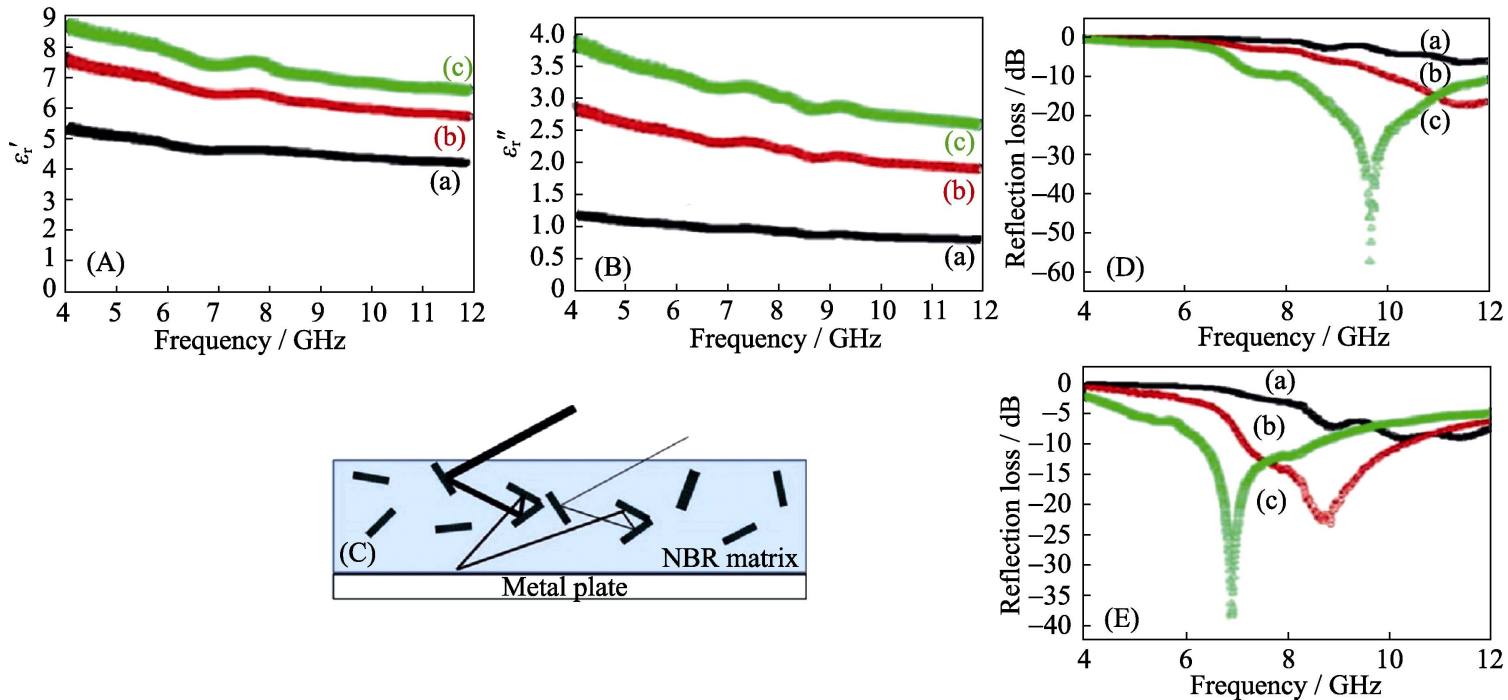

图 $3 \mathrm{rGO}$ /丁晴橡胶的介电常数与反射率曲线

Fig. 3 Complex permittivity and reflection loss curves of rGO/NBR composites

(A) Real part of permittivity $\left(\varepsilon^{\prime}\right)^{[38]}$; (B) Imaginary part of permittivity $\left(\varepsilon^{\prime \prime}\right)^{[38]}$; (C) A possible absorption mechanism; (D-E) Reflection loss characteristics with thickness: (D) $3 \mathrm{~mm}$ and (E) $4 \mathrm{~mm}$ for rGO/NBR composites ${ }^{[38]}$

(a) $2 \mathrm{wt} \% \mathrm{rGO}$; (b) $4 \mathrm{wt} \% \mathrm{rGO}$; (c) $10 \mathrm{wt} \% \mathrm{rGO}$ 
管/石墨烯在层厚为 $2 \mathrm{~mm}$ 时最大反射损耗为 $-18.9 \mathrm{~dB}^{[41]}$ 。多壁碳纳米管由于缺陷多和导电性优 良，从而具有更好的吸波性能，其与石墨烯复合最 大反射损耗为 $-39.5 \mathrm{~dB}$, 且在整个 $\mathrm{C}$ 波段 (4 8 $\mathrm{GHz})$ 和 $\mathrm{X}$ 波段 $\left(8 \sim 12 \mathrm{GHz}\right.$ )的反射损耗均低于 $-22.5 \mathrm{~dB}^{[42]}$ 。

(2) 石墨烯/纳米金属(及其化合物)复合材料

纳米金属是良好的吸波材料, 具有宽频带和兼 容性好等特点。根据能带理论, 减小颗粒尺寸易形 成不连续能级, 分裂后的能级间隔处于微波能量范 围内，从而产生新的吸波通道增强吸波性能。磁性 金属微粉是一类非常重要的纳米金属电磁波吸收剂, 但在使用中也存在易氧化、耐腐蚀能力差、密度较 大和低频段吸收性能差等问题。因此, 减小粒径, 进 行表面改性、掺杂或包覆是该类材料的主要发展方 向。石墨烯的大比表面积有利于改善材料间的相互 接触，提供良好的电子传输通道，其与金属纳米材 料复合可增强界面极化强度，从而提高高频介电损 耗，增强吸波效果。

通过掺杂 $\mathrm{Fe}^{[43]} 、 \mathrm{Ni}^{[44-47]} 、 \mathrm{Co}^{[48-49]}$ 等粒子能提 高石墨烯基复合材料对电磁波的损耗能力 ${ }^{[50]}$ 。改变 反应条件, $\mathrm{Ni} 、 \mathrm{Co}$ 粒子可以从密排六方晶体结构向 面心立方晶体结构转变 ${ }^{[46,}{ }^{48]}$, 研究发现, 密排六方 结构 $\mathrm{Co} /$ 石墨烯电磁波损耗能力优于立方面心晶体 结构 $\mathrm{Co}$ /石墨稀。

纳米金属氧化物 $\left(\mathrm{Co}_{3} \mathrm{O}_{4}{ }^{[51-52]} 、 \mathrm{ZnO}^{[53-58]}\right.$ 、 $\mathrm{SrTiO}_{3}{ }^{[59]} 、 \mathrm{NiO}^{[60-61]}$ 和 $\left.\mathrm{MgO}^{[62]}\right)$ 、纳米金属粒子 $\left(\mathrm{CoS}_{2}{ }^{[63]} 、 \mathrm{NiCO}_{2}{ }^{[64]} 、 \mathrm{MoS}_{2}{ }^{[65]}\right.$ )、纳米金属片(层状 $\mathrm{CuS}^{[66]}$ 、层状 $\mathrm{FeCoB}^{[67]}$ )、层状羰基铁 ${ }^{[68-69]}(\mathrm{FCI})$ 和
金属有机框架化合物 $(\mathrm{MOF})^{[70]}$ 与石墨烯复合大大提 升了对电磁波的损耗能力。通过调控反应条件可进 一步提高产物对电磁波的损耗，如 $\mathrm{Co}\left(\mathrm{NO}_{3}\right)_{2} \cdot 6 \mathrm{H}_{2} \mathrm{O}$ 与氧化石墨烯发生还原反应, 实现生成物由 $\mathrm{Co}_{3} \mathrm{O}_{4}$ 向 $\mathrm{Co}$ 的转变 ${ }^{[51,71]}$, 当产物为 $\mathrm{Co}_{3} \mathrm{O}_{4} / \mathrm{Co} /$ 石墨烯时最 大反射损耗达 $-52.8 \mathrm{~dB}$; 优化反应物结构或石墨烯

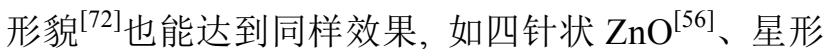
$\mathrm{ZnO}^{[57]}$ 或空心 $\mathrm{ZnO}$ 多孔微球 ${ }^{[58]}$ 分别与石墨烯复合, 所得材料最大反射损耗值达 $-77.5 \mathrm{~dB}$ 。图 4 为用于石 墨烯复合的部分典型纳米金属化合物的结构形貌。

(3) 石墨烯/铁氧体复合材料

铁氧体属于双复介电材料, 既有亚铁磁性又有 介电特性。虽然铁氧体吸波材料具有吸收性能优异、 成本低廉等优点, 但纯铁氧体由于密度较大、高温 表现欠佳和吸收频带窄等缺点难以满足高性能吸波 材料要求，需将铁氧体粉末分散到非磁性微粒中制 成复合铁氧体材料来改善产物吸波性能。将铁氧体 与石墨烯复合可以发挥各自优点, 通过多种损耗途 径吸收电磁波，达到密度小且高效吸收的效果。

Luo 等 ${ }^{[73]}$ 通过计算模拟预测石蜡基片状羰基铁 粉/蜂窝状石墨烯的最大反射损耗可达 $-27.5 \mathrm{~dB}$, $8 \sim 12 \mathrm{GHz}$ 时全频段反射损耗低于 $-10 \mathrm{~dB}$, 且蜂窝 状石墨烯对电磁波的损耗起到非常重要的作用。由 此可见, 改变基体材料种类、反应物结构及各组分 间相互结合状态可以提高复合材料吸波性能, $\mathrm{Fe}_{3} \mathrm{O}_{4} /$ 石墨烯复合材料体系存在类似的情况。虽然 $\mathrm{Fe}_{3} \mathrm{O}_{4} /$ 石墨烯最大反射损耗仅为-22.7 $\mathrm{dB}^{[74-75]}$, 但通过改 善 $\mathrm{Fe}_{3} \mathrm{O}_{4}$ 在石墨烯中的分散性 ${ }^{[76-77]}$, 改变 $\mathrm{Fe}_{3} \mathrm{O}_{4}{ }^{[78-80]}$ (a)

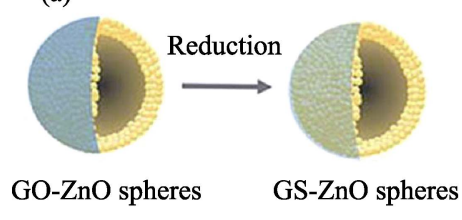

(d)

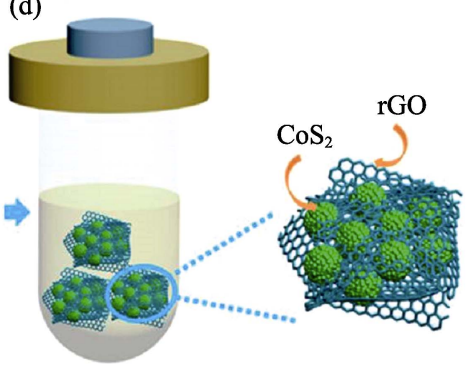

(b)

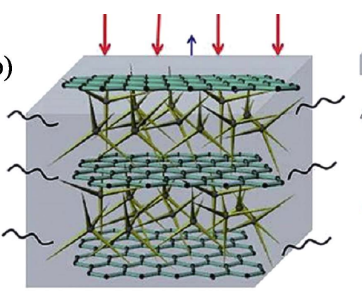

Paraffin

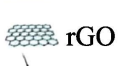

$\chi \mathrm{T}-\mathrm{ZnO}$

$\longrightarrow$ Incident microwave

$\longrightarrow$ Reflection

$\sim$ Heat

(c)

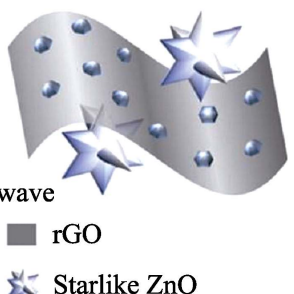

站 Starlike $\mathrm{ZnO}$

图 4 用于石墨烯复合的部分典型纳米金属化合物的结构形貌

Fig. 4 Typical microstructures of metal nanomaterials for graphene composites (a) $\mathrm{ZnO}$ spheres ${ }^{[55]}$; (b) $\mathrm{T}-\mathrm{ZnO}^{[56]}$; (c) Starlike- $\mathrm{ZnO}^{[57]}$; (d) $\mathrm{CoS}_{2}$ spheres and reflection loss curves ${ }^{[63]}$ 
(胶囊状 $\mathrm{Fe}_{3} \mathrm{O}_{4}$ 、团簇状 $\mathrm{Fe}_{3} \mathrm{O}_{4}$ 、亚微球状 $\mathrm{Fe}_{3} \mathrm{O}_{4}$ 和碳包 覆顺磁性 $\mathrm{Fe}_{3} \mathrm{O}_{4}$ ) 或石墨烯形貌结构 ${ }^{[81-82]}$ 可以增强材 料对电磁波的损耗。调控反应条件(如提升反应温 度), 会发生由 $\alpha-\mathrm{Fe}_{2} \mathrm{O}_{3}$ 向 $\gamma-\mathrm{Fe}_{2} \mathrm{O}_{3}$ 再向 $\mathrm{Fe}_{3} \mathrm{O}_{4}$ 晶体结 构的转变过程 ${ }^{[83]}$, 晶体结构的改变对不同结构铁的 氧化物/石墨烯复合材料电磁波损耗能力影响极大。 枸杞状 $\alpha-\mathrm{Fe}_{2} \mathrm{O}_{3}$ /石墨烯 ${ }^{[84]}$ 在 $5.6 \mathrm{GHz}$ 时最大反射损 耗为 $-46.6 \mathrm{~dB}$, 经表面修饰的团簇状纳米胶体 $\gamma-\mathrm{Fe}_{2} \mathrm{O}_{3}$ /石墨烯 ${ }^{[85]}$ 最大反射损耗为 $-59.65 \mathrm{~dB}$ 。对 $\alpha-\mathrm{Fe}_{2} \mathrm{O}_{3}$ 晶体结构进行修饰, 可得空心纳米棒状 $\alpha-\mathrm{Fe}_{2} \mathrm{O}_{3}$ /石墨烯复合材料 ${ }^{[86]}$, 其最大反射损耗为 $-71.4 \mathrm{~dB}$, 在 $\mathrm{Fe}_{2} \mathrm{O}_{3}$ /石墨烯复合材料中添加 $\mathrm{TiO}_{2}{ }^{[87]}$ 、 $\mathrm{SnO}_{2}{ }^{[88]}$ 都能提升材料对电磁波的损耗能力。

$\mathrm{BiFeO}_{3}$ 纳米线 ${ }^{[89]} 、 \mathrm{MgFe}_{2} \mathrm{O}_{4}$ 纳米棒 ${ }^{[00]}$ 、 $\mathrm{MnFe}_{2} \mathrm{O}_{4}{ }^{[91]} 、 \mathrm{BaFe}_{12} \mathrm{O}_{19}{ }^{[92]} 、 \mathrm{CoFe}_{2} \mathrm{O}_{4}{ }^{[93-95]} 、 \mathrm{BaTiO}_{3}{ }^{[96]}$ 、 $\mathrm{Bi}_{2} \mathrm{Fe}_{4} \mathrm{O}_{9}{ }^{[97]} 、 \mathrm{Ni}_{0.5} \mathrm{Zn}_{0.5} \mathrm{Fe}_{2} \mathrm{O}_{4}{ }^{[98]}$ 和 $\mathrm{NiFe}_{2} \mathrm{O}_{4}{ }^{[99-102]}$ 等 与石墨烯复合都有良好的电磁波损耗效果, 通过调 控石墨烯形态及 $\mathrm{Bi}_{2} \mathrm{Fe}_{4} \mathrm{O}_{9}$ 结构 ${ }^{[97]}$, 可以获得最大反 射损耗为 $-71.88 \mathrm{~dB}$ 的多孔 $\mathrm{Bi}_{2} \mathrm{Fe}_{4} \mathrm{O}_{9}$ 微球/石墨烯 复合材料。用氢气/氨气混合气氛对 $\mathrm{CoFe}_{2} \mathrm{O}_{4}$ /石墨 烯还原得到 $\mathrm{FeCo}$ /石墨烯复合材料, $8.9 \mathrm{GHz}$ 时最大 反射损耗为 $-40.2 \mathrm{~dB}^{[95]}$ 。

\section{(4) 石墨烯/导电聚合物复合材料}

导电聚合物具有密度低、结构多样和环境适应 性好等优点, 经掺杂修饰后的导电聚合物复合材料 的导电性具有在绝缘体、半导体和金属性范围内变 化的特性, 因此可通过控制导电聚合物电导率调节 其电磁波损耗能力。利用石墨烯修饰导电聚合物, 可以有效改善产物阻抗匹配性, 降低层厚度, 使电 导率可调, 从而达到最优吸波效果。

常见制备石墨烯一导电聚合物纳米复合材料的 基体包括: 聚吡咯、丙烯腈丁二烯、聚甲基丙烯酸 甲酯、聚苯胺和环氧乙烷等。其中, 聚环氧乙烷/石 墨烯的最大反射损耗为 $-38.8 \mathrm{~dB}^{[39]}$, 多孔丙烯腈丁 二烯橡胶/氧化石墨烯的最大反射损耗为 $-57 \mathrm{~dB}^{[38]}$ 。 6 层聚甲基丙烯酸甲酯/石墨烯在 $30 \mathrm{GHz}$ 左右电磁 波损耗效果最佳 ${ }^{[103]}$ 。

(5) 石墨烯/非金属复合材料

由于硼、氮原子与碳原子结构相似, 相互结合 可形成 $n$-型或 $p$-型半导体, 且通过调节硼、氮和碳 原子组成比例可以获得一系列电磁参数可调的 B-C-N 吸波材料 ${ }^{[104-105]}$ 。研究显示掺氮石墨烯中吡 咯氮位可诱导材料磁损耗, 在 $12.7 \mathrm{GHz}$ 时最大反射 损耗为 $-11.3 \mathrm{~dB}^{[106]}$ 。掺杂碳纤维的 $\mathrm{BCN}$ 复合材料 在 $9.41 \mathrm{GHz}$ 时最大反射损耗为 $-29.23 \mathrm{~dB}^{[107]}$ 。纳米 $\mathrm{BNNC} /$ 石墨烯的最大反射损耗达 $-32.38 \mathrm{~dB}$, 且当层
厚为 $1.5 \sim 5.0 \mathrm{~mm}$ 时，有效反射频宽为 $3.49 \sim$ $17.28 \mathrm{GHz}^{[105]}$ 。

本课题组利用硼酸、尿素及氨嗍烷和氧化石墨 烯反应制备 h-BN/石墨烯复合材料 ${ }^{[108-109]}$, 测量发 现，面内掺杂的 $\mathrm{BCN}$ 材料最大反射损耗为 $-33.6 \mathrm{~dB}$, 层间掺杂的 $\mathrm{BCN}$ 材料最大反射损耗为 $-40.5 \mathrm{~dB}$ 。

\subsubsection{2 石墨烯基三元复合材料}

相较石墨烯基二元复合材料, 三元及以上多元 组分复合可进一步改善产物阻抗匹配性, 提升电磁 波损耗能力。目前，三元复合材料主要以介电损耗 和磁损耗协同消耗机理为主。其中, 石墨烯较大的 比表面积在复合材料中可以形成导电网络, 载流子 受电磁波激发在导电网络中取向运动从而产生介电 损耗; 加载磁性材料且通过调控负载的密度、形貌、 结构和各组元含量, 增加复合材料磁损耗; 随着组 元的增多, 各组元材料在复介电常数上的差值导致 的界面散射, 加剧了材料对电磁波的损耗作用。故 需将不同形貌和结构的铁氧体、纳米金属、导电聚 合物及纳米碳材料与石墨烯结合, 制备集吸波和承 载于一体的具有优异化学、力学和电学性能的高效 电磁波损耗材料 ${ }^{[110-115]}$ 。

通过引入碳纳米管 ${ }^{[116-120]}$ (沙棘状单壁碳纳米 管 ${ }^{[119]}$ 、多壁碳纳米管 ${ }^{[120]}$ 、纳米棒状 $\mathrm{ZnO}^{[121]}$ 、片 层 $\mathrm{MoS}_{2}{ }^{[122]}$ 可以有效阻止 $\mathrm{Fe}_{3} \mathrm{O}_{4} /$ 石墨烯复合材料中 各组分团聚, 提高产物吸波性能。经磁性中空 $\mathrm{CoFe}_{2} \mathrm{O}_{4}$ 微粒修饰的多壁碳纳米管与石墨烯的复合 材料的最大反射损耗达 $-46.8 \mathrm{~dB}$, 有效反射损耗频 宽为 $13.1 \mathrm{GHz}^{[123]}$ 。 $\mathrm{Co}_{3} \mathrm{O}_{4}$ 和氧化石墨烯反应可制备 经 Co 离子催化原位生成碳纳米管的 Co@碳纳米管/ 石墨烯复合材料 ${ }^{[124]}$, 通过调节反应温度, 调控碳纳 米管生长含量, 实现对不同频率电磁波的损耗。当 反应温度分别为 400 和 $450^{\circ} \mathrm{C}$ 时, 最大反射损耗可 达 -65.6 和 $-58.1 \mathrm{~dB}$ 。

用氧酸酯树脂 ${ }^{[125]}$ 或聚(对亚苯基苯并二恶

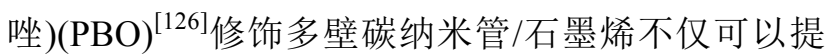
升产物吸波性能, 还可以提高其耐高温特性。用聚 乙烯吡咯烷酮修饰的片状羰基铁/石墨烯 ${ }^{[127]}$ 最大反 射损耗为 $-27.59 \mathrm{~dB}$, 将片状羰基铁研磨成粉可大幅 提升材料对电磁损耗能力 ${ }^{[128]}$ 。用环氧树脂代替聚乙 烯吡咯烷酮制备环氧树脂/片状羰基铁/石墨烯最大 反射损耗值为 $-32.3 \mathrm{~dB}^{[129]}$ 。二元组分聚苯胺/石墨烯 复合材料 ${ }^{[130-132]}$ 的最大反射损耗为 $-45.1 \mathrm{~dB}$ 。用 $\mathrm{Cu}_{2} \mathrm{O}^{[133]} 、 \mathrm{Co}_{3} \mathrm{O}_{4}{ }^{[134]} 、 \mathrm{CoFe}_{2} \mathrm{O}_{4}{ }^{[135]} 、 \mathrm{NiFe}_{2} \mathrm{O}_{4}{ }^{[136]} 、$ $\mathrm{CuFe}_{10} \mathrm{Al}_{2} \mathrm{O}_{19}{ }^{[137]}$ 或 $\mathrm{CNT}-\mathrm{Fe}_{3} \mathrm{O}_{4}{ }^{[138]}$ 改性可有效提高 材料层间阻抗匹配性, 增强界面极化作用。其中, 
$\mathrm{CuFe}_{10} \mathrm{Al}_{2} \mathrm{O}_{19}$ /石墨烯/聚苯胺电磁波损耗能力最优, 最大反射损耗达到-63.6 dB。经棒状聚苯胺修饰的 $\mathrm{Fe}_{3} \mathrm{O}_{4} / \mathrm{C} /$ 石 墨烯复合材料 ${ }^{[139]}$ 中棒状聚苯胺是否共 价连接对其吸波性能影响极大, 共价键合聚苯胺修 饰的复合材料吸波性能远优于非共价键修饰效果。 经测试所得材料在层厚 $3 \mathrm{~mm} 、 11.4 \mathrm{GHz}$ 时, 最大反 射损耗为 $-44.2 \mathrm{~dB}$ 。

\section{2 三维结构石墨烯电磁波损耗材料}

\subsection{1 “核一壳” 结构石墨烯基复合材料}

“核一壳”结构复合材料是通过化学键或其它相 互作用包覆形成的有序组装结构, 其核心原子、外 壳原子以及可能存在的中间层或空隙层之间的电子 交换使整个材料性能发生质变, 从而改善不同组分 原有的缺陷，提高材料对电磁波的损耗能力。

石墨烯修饰的 “核一壳” 结构复合材料电磁波损 耗能力优异, 多孔碳纳米微球@FeNi/石墨烯最大 反射损耗为-45.2 $\mathrm{dB}^{[140]}, \mathrm{CoNi} @ \mathrm{SiO}_{2}$ /石墨烯最大反 射损耗达到 $-50.3 \mathrm{~dB}^{[141]}$ 。铁氧体/石墨烯复合材料 (中空结构 $\mathrm{Fe}_{3} \mathrm{O}_{4} @ \mathrm{Fe} /$ 石墨烯 ${ }^{[142]}$ 、纳米棒状 $\mathrm{SiO}_{2} @ \mathrm{Fe}_{3} \mathrm{O}_{4} /$ 石墨烯 ${ }^{[143]}$ 、多孔状 $\mathrm{ZnO} @ \mathrm{p}-\mathrm{Fe}_{3} \mathrm{O}_{4} /$ 石墨 烯 ${ }^{[120]} 、 \mathrm{FeCo} @ \mathrm{SiO}_{2}$ /石墨烯 ${ }^{[144]} 、 \mathrm{ZnFe}_{2} \mathrm{O}_{4} @ \mathrm{SiO}_{2} /$ 石 墨烯 ${ }^{[145]}$ 及 $\mathrm{MoS}_{2} @ \mathrm{Fe}_{3} \mathrm{O}_{4}$ 石墨烯 ${ }^{[122]}$ )不仅电磁波损 耗能力优异, 而且实现了材料轻量化要求。“核一壳” 结构 $\mathrm{Fe}_{3} \mathrm{O}_{4} @$ 聚吡咯/石墨烯三元复合材料 ${ }^{[146]}$ 最大 反射损耗为 $-25.1 \mathrm{~dB}$, 通过调控聚吡咯形态, 如树 枝状聚吡咯/ $\mathrm{CoFe}_{2} \mathrm{O}_{4} /$ 石墨烯 ${ }^{[147]}$ 和聚吡咯纳米棒/石 墨烯复合材料 ${ }^{[148]}$ 可以具有更加优异的电磁波损耗 能力。

“核一壳”结构 $\mathrm{CoNi} @ \mathrm{SiO}_{2} @$ 石墨烯一聚苯胺四 元复合材料的最大反射损耗达 $-43 \mathrm{~dB}^{[149]}$ 。掺杂 “核一壳-壳” 结构 $\mathrm{Fe}_{3} \mathrm{O}_{4} @ \mathrm{SiO}_{2} @$ 聚苯胺的石墨烯 复合材料的最大反射损耗为 $-40.7 \mathrm{~dB}^{[150]}$, 用聚苯胺 替换 $\mathrm{NiO}$, 可以制备多层包覆结构的石墨烯 $@ \mathrm{Fe}_{3} \mathrm{O}_{4} @ \mathrm{SiO}_{2} @ \mathrm{NiO}$ 复合材料 ${ }^{[151]}$, 当厚度为 $1.8 \mathrm{~mm} 、 14.6 \mathrm{GHz}$ 时, 最大反射损耗 $-51.5 \mathrm{~dB}$ 。 $\mathrm{Fe}_{3} \mathrm{O}_{4} @ \mathrm{Fe} / \mathrm{ZnO} /$ 石墨烯四元复合材料 ${ }^{[152]}$ 在层厚为 $2.5 \sim 5 \mathrm{~mm}$ 时, 最大反射损耗均低于 $-30 \mathrm{~dB}$, 且反 射损耗值低于 $-20 \mathrm{~dB}$ 的频宽为 $7.3 \mathrm{GHz}$ 。

\subsection{2 “三明治”结构石墨烯基复合材料}

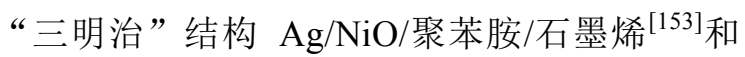
$\mathrm{Fe}_{3} \mathrm{O}_{4} / \mathrm{WO}_{3} /$ 聚苯胺/石墨烯 ${ }^{[154]}$ 由于介电损耗和磁损 耗的协同作用, 电磁损耗能力优异。利用碳纳米管 修饰的 $\mathrm{BaFe}_{12} \mathrm{O}_{19}$ /三维石墨烯 ${ }^{[155]}$ 最大反射损耗值 为 $-18.35 \mathrm{~dB}$ 。而掺杂 $\mathrm{Ni}$ 的石墨烯/C 在 $1.6 \mathrm{~mm}$ 、 $13.9 \mathrm{GHz}$ 时, 最大反射损耗为 $-34.2 \mathrm{~dB}^{[156]}$ 。掺杂 $\mathrm{ZnO}$ 的 $\mathrm{CoO} @ \mathrm{C} /$ 石墨烯在层厚 $2.6 \mathrm{~mm}, 11.3 \mathrm{GHz}$ 时最
大反射损耗达 $-51.1 \mathrm{~dB}$, 有效反射频宽为 $4.7 \mathrm{GHz}^{[157]}$ 。

\subsection{3 “多孔泡沫” 结构石墨烯基复合材料}

微观结构的变化可以显著改善材料吸波性能,

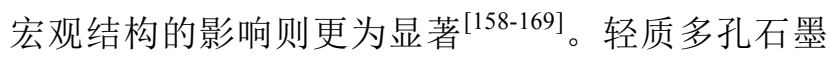
烯泡沫在 2 110 GHz 范围内吸波效果良好, 且可通 过简单物理压缩对电磁波损耗实现调控。研究发现 经不同浓度反应溶液和处理温度制备的石墨烯泡沫 吸波性能与微结构中的碳/氧比例和共轭碳畴尺寸 密切相关。

掺杂 $\mathrm{Ni}$ 粒子的多孔石墨烯发泡材料最大反射 损耗达-59.7 $\mathrm{dB}^{[170]}$; 一维纳米金属线 $(\mathrm{ZnO}$ 纳米 线 ${ }^{[171]} 、 \mathrm{Si}_{3} \mathrm{~N}_{4}$ 纳米线、 $\mathrm{SiC}$ 纳米线 ${ }^{[172-173]}$ )/石墨烯复 合材料吸波性能优异且高温条件下材料结构保持长 久稳定 (质量损失低于 $10 \%$ ); 掺杂 $\mathrm{TiO}_{2}{ }^{[174]}$ 、 $\mathrm{Mn}_{3} \mathrm{O}_{4}{ }^{[175]}$ 和 $\mathrm{Fe}_{3} \mathrm{O}_{4}{ }^{[176]}$ 的石墨烯气凝胶具有轻质和 反射耗散强等特点; $\mathrm{Al}_{2} \mathrm{O}_{3}{ }^{[177]} 、 \mathrm{SiCN}^{[178]}$ 与石墨烯烧 结可合成具有优良力学性能及良好电磁波损耗特性 的陶瓷材料, 这为新材料在军事装备上的应用奠定 了良好的基础。

\section{4 吸波性能的影响因素}

纯石墨烯材料多采用化学氧化还原法制备, 大 量存在的缺陷及残留的含氧基团不仅可以改善石墨 烯的阻抗匹配性, 还可以有效提高其吸波性能。通 过水/溶剂热法、共沉淀法、溶胶一凝胶法、原位聚 合溶液共混法、熔融共混法及冷冻干燥法可以制备 功能化的石墨烯基复合材料, 制备过程要求尽可能 减小晶体颗粒尺寸，提高不同组分之间的分散性， 使得反应过程容易控制, 从而通过掺杂/改性达到增 强石墨烯基复合材料电磁波损耗能力的作用 ${ }^{[179-187]}$ 。

目前，基于石墨烯的吸波材料：在制备方法上， 二元复合材料多采用一步法, 三元复合材料多采用 两步法; 在结构上, 三维多孔结构逐渐受到人们的 重视; 在复合基材上, 趋向于选用多元的不同吸波 机理的吸波基材。下面分别从损耗机制、组分数量 及微观结构三个方面对石墨烯基电磁波损耗材料吸 波性能的影响因素进行分析。图 5 为典型石墨烯基 电磁波损耗材料最大反射率值与厚度的关系。

\section{1 损耗机制影响因素}

纯石墨烯是介电损耗型吸波材料, 引入吡咯位 氮原子可诱发产生磁损耗，而将铁氧体材料、金属 粒子或金属化合物与石墨烯通过单一或与多元组分 搭配复合可进一步提升产物电磁波损耗能力(如图 5 所示)。这主要得益于多元组分间良好的阻抗匹配性及 介电损耗和磁损耗的协同消耗机理。例如, Yuan 等 ${ }^{[47]}$ 

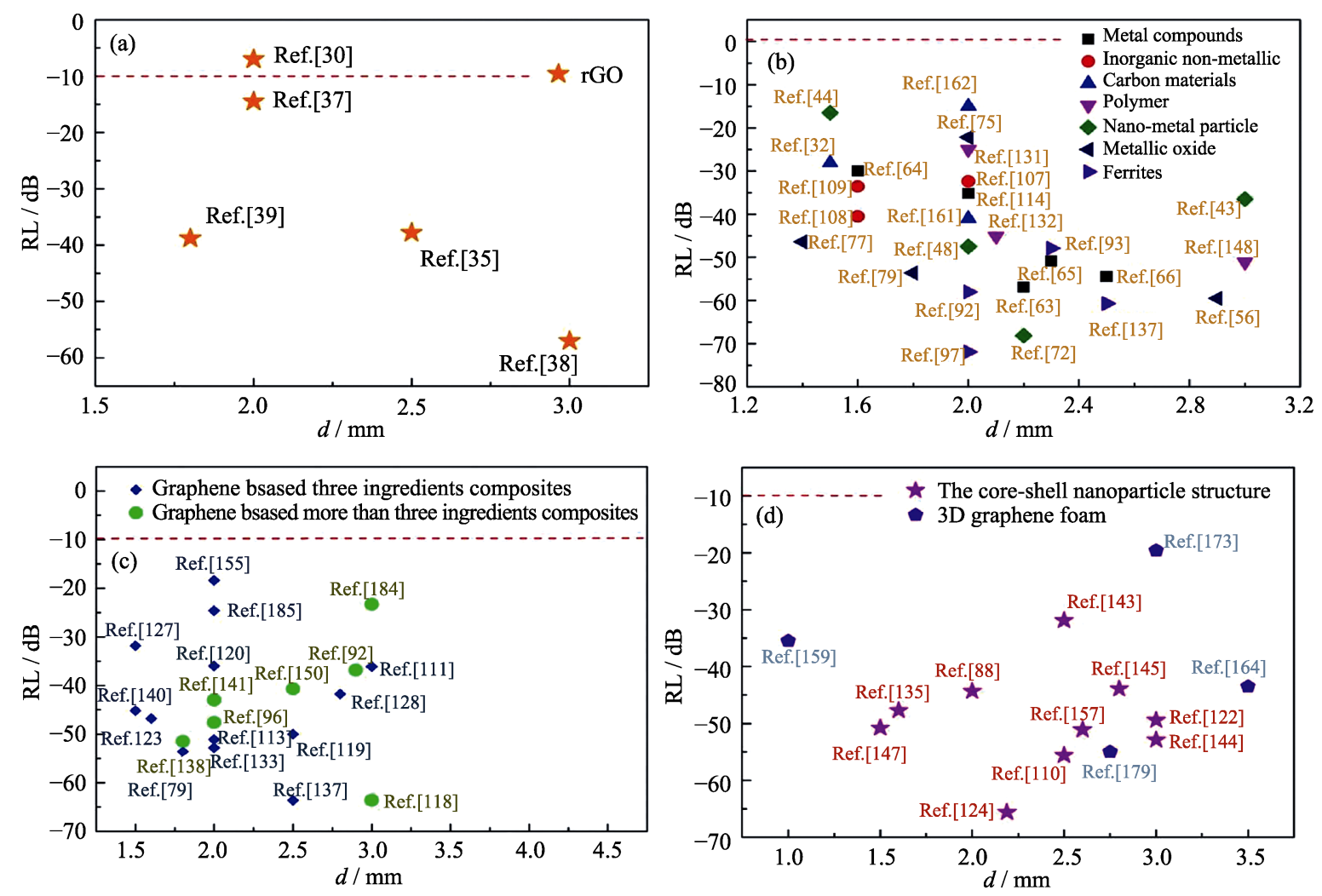

图 5 石墨烯基电磁波损耗材料最大反射率值与厚度的关系

Fig. 5 Schematic of relationship between maximum reflectivity and thickness of graphene based electromagnetic wave loss material

(a) rGO; (b) Two ingredients; (c) Three or more ingredients; (d) Three-dimensional structure

制备的 $\mathrm{Ni}$ 粒子掺杂的 $\mathrm{N}$-石墨烯复合材料, 在 $2 \sim 18 \mathrm{GHz}$ 频率范围内, 介电损耗角正切值 $\left(\tan \delta_{\mathrm{e}}\right)$ 均大于 0.4 , 说明介电损耗发生在所有频率范围内，同时磁损耗 角正切值 $\left(\tan \delta_{\mathrm{m}}\right)$ 均小于 0.4 , 表明磁损耗小于介电损 耗，即所合成复合材料主要依赖于介电损耗。再如， Zhou 等 ${ }^{[141]}$ 合成的 $\mathrm{CoNi} @ \mathrm{SiO}_{2} @ \mathrm{RGO}$ 复合材料, 在 石墨烯中加入 $\mathrm{CoNi} @ \mathrm{SiO}_{2}$ 后电导率降低, 使得介电 常数的实部和虚部值降低, 介电损耗减小, 但增强 了复合材料阻抗匹配性。在 2 18 GHz 频率范围内, 介电损耗角正切值 $\left(\tan \delta_{\mathrm{e}}\right)$ 均小于 0.4 , 但在约 $17 \mathrm{GHz}$ 左右时出现最高峰。同时磁损耗角正切值 $\left(\tan \delta_{\mathrm{m}}\right)$ 在 $0 \sim 16 \mathrm{GHz}$ 均大于 0 , 说明磁损耗发生在所有频率范 围内, 在约 $9 \mathrm{GHz}$ 时出现最高峰且大于同频率下的 介电损耗角正切值, 表明 $\mathrm{CoNi} @ \mathrm{SiO}_{2} @ \mathrm{RGO}$ 复合材 料在介电损耗和磁损耗协同作用下增加了对电磁波 的损耗。

\section{2 组分数量影响因素}

由图 5 可知, 二维结构石墨烯复合材料中, 三元/ 多元石墨烯基电磁波损耗材料的电磁波损耗能力整 体优于二元复合材料, 这是由于多层材料各组分间 良好的阻抗匹配性，增强的界面/层间极化效应、介 电损耗和磁损耗相互协同效应及粒子间的空隙对电
磁波的折/反射效应叠加所致。但组分过多会造成材 料密度增大、反应过程难以精确控制、基材分散不 均匀、界面相容性差等问题。因此石墨烯基电磁波 损耗材料应以不多于三组分组合为主。

\section{3 结构影响因素}

无论是改变反应条件, 还是各组分结合状态, 都会促使产物形貌及微观结构发生变化。研究发现, 形貌和结构的差异对复合材料电磁波损耗能力影响 很大。通过调控产物形貌及结构, 改变阻抗匹配性, 增加界面及层间极化作用, 可以提高复合材料吸波 性能。如 Chen 等 ${ }^{[31]}$ 通过对微结构的设计制备了一 种 “花朵” 结构的多孔石墨烯材料, 通过利用 3D 多 孔褶皱石墨烯结构构建 3D 导电网络, 对微波实现 多重反射, 从而大幅提升材料对电磁波的损耗能力, 使最大反射损耗达到 $-42.9 \mathrm{~dB}$ 。Zhang 等 ${ }^{[158]}$ 将石墨 烯发泡制备多孔石墨烯泡沫，所得材料在全波段都 具有非常优异的吸波性能。最近研究发现 3D 石墨 烯结构对 THz 频段也具有良好的损耗作用 ${ }^{[188]}$ 。

综上所述, 三维结构石墨烯基复合材料较二维 结构而言对电磁波有更大的损耗途径及折/反射空 间, 且兼具轻质和耐高温等特性, 从而成为石墨烯 基电磁波损耗材料的重要发展方向。 
表 1 典型石墨烯基电磁波损耗材料性能统计表

Table 1 Comparison of EMW absorption properties of the materials based on graphene

\begin{tabular}{|c|c|c|c|c|c|}
\hline Filler & Matrix & $\begin{array}{l}\text { Reflection } \\
\text { Loss/dB }\end{array}$ & $\begin{array}{l}\text { Optimum } \\
\text { thickness/mm }\end{array}$ & $\begin{array}{l}\text { Effective bandwidth in } \\
2-18 \mathrm{GHz} / \mathrm{GHz}\end{array}$ & Ref. \\
\hline rGO & Paraffin & -7.0 & 2.0 & - & {$[30]$} \\
\hline rGO & PEO & -38.8 & 1.8 & 4.10 & [39] \\
\hline Pitch carbon coating grapheme/carbon nanotubes & Paraffin & -18.9 & 2.0 & $\sim 4.0$ & [41] \\
\hline $\mathrm{rGO} /$ polyaniline & Paraffin & -25.0 & 2.0 & 4.0 & {$[131]$} \\
\hline rGO-BN & Paraffin & -40.5 & 1.6 & 5.0 & [108] \\
\hline$\alpha$-cubic $\mathrm{Co} / \mathrm{GN}$ & Paraffin & -47.5 & 2.0 & 5.3 & [48] \\
\hline $\mathrm{Fe}_{3} \mathrm{O}_{4} / \mathrm{N}$-graphene & Paraffin & -53.6 & 1.8 & 5.0 & {$[79]$} \\
\hline Tetrapod-like $\mathrm{ZnO} / \mathrm{rGO}$ & Paraffin & -59.5 & 2.9 & 6.9 & {$[56]$} \\
\hline $\mathrm{MCI} / \mathrm{rGO} / \mathrm{PVP}$ & Paraffin & -41.7 & 2.8 & 12.52 & [128] \\
\hline $\mathrm{rGO} / \mathrm{CoFe}_{2} \mathrm{O}_{4} / \mathrm{MWCNT}$ & Paraffin & -46.8 & 1.6 & 3.44 & {$[123]$} \\
\hline $\mathrm{Gr} / \mathrm{Ti} @ \mathrm{CNT} / \mathrm{Fe}_{3} \mathrm{O}_{4} / \mathrm{PANI}$ & TPU & -63.57 & 3.0 & 4.20 & [118] \\
\hline $\begin{array}{c}\text { Yolk-shell CoO@Co } \\
\text { NPs/ZnO NPs/graphene }\end{array}$ & Paraffin & -51.1 & 2.6 & 4.70 & [157] \\
\hline rGO foams & - & -33.2 & 1.0 & $\sim 14.00$ & {$[158]$} \\
\hline 3D-rGO/silica textile/PF & - & -36.0 & 3.3 & 4.20 & {$[160]$} \\
\hline Porous graphene microflowers & Paraffin & -42.8 & 2.0 & 5.59 & {$[31]$} \\
\hline
\end{tabular}

\section{5 结束语}

本文系统地总结了近几年来在石墨烯基电磁波 损耗复合材料方面的工作, 其中典型石墨烯基电磁 波损耗复合材料吸波性能如表 1 所示。目前的研究 已经证明石墨烯以其独特的物理结构、优异的力学 和电学性能, 在改善复合材料不同组分间的阻抗匹 配性，提高材料电磁波损耗能力等方面有着巨大的 应用潜力。但纯石墨烯材料作为介电损耗型吸波剂, 对电磁波损耗效果欠佳, 需要对其结构进行优化, 同时与其它物质复合, 从而可以制备轻质、高效且 力学性能优异的电磁波损耗材料。总之, 石墨烯基 电磁波损耗材料得到了科学界的广泛关注, 作为新 型吸波材料已成为未来应用的研究重点, 但其在以 下几个研究方向仍存在一些挑战, 值得进一步深入 研究:

1)多组分石墨烯基电磁波损耗材料吸波机理 研究;

2)三维复合结构石墨烯基电磁波损耗材料 研究;

3)石墨烯基电磁波损耗材料功能与结构一体化 设计研究。

为了进一步提升石墨烯基电磁波损耗材料的吸 波性能，尽快在相关领域开展应用。必须开发规模 化、量产化的石墨烯制备技术和复合工艺，提高石 墨烯质量。在减重和增效基础上实现功能与结构一 体化设计目标，这不仅会推动军事隐形材料的发
展，还会在防护电磁波辐射等民用方面发挥重要 作用。

\section{参考文献:}

[1] KROTO H W, HEATH J R, OBRIEN, S C, et al. C60: Buckminsterfullerene. Nature, 1985, 318(6042): 162-163.

[2] IIJIMA S. Helical microtubules of graphitic carbon. Nature, 1991, 354(6348): 56-58.

[3] GEIM A K, NOVOSELOV K S. The rise of graphene. Nature Materials, 2007, 6(3): 183-191.

[4] NOVOSELOV K S, GEIM A K, MOROZOV S V, et al. Electric field effect in atomically thin carbon films. Science, 2004, 306(5696): 666-669.

[5] KIN K, ZHAO Y, JANG H, et al. Large-scale pattern growth of graphene films for stretchable transparent electrodes. Nature, 2009, 457(7230): 706-710.

[6] GEIM K. Graphene: status and prospects. Science, 2009, 324(5934): 1530-1534.

[7] DMITRY V, KOSYNKINL A L, HIGGINBOTHAM J, et al. Longitudinal unzipping of carbon nanotubes to form graphene nanoribbons. Nature, 2009, 458(7240): 872-876.

[8] PAN Y, ZHANG H, SHI D, et al. Highly ordered, millimeter-scale, continuous, single crystalline graphene monolayer formed on $\mathrm{Ru}(0001)$. Advanced Materials, 2009, 21(27): 2777-2780.

[9] CHOI W B, LAHIRI I, SEELABOYINA R. Synthesis of graphene and its applications: a review. Critical Reviews in Solid State and Materials Sciences, 2010, 35(1): 52-71.

[10] ZHU Y W, MURALI S T, CAI W W, et al. Graphene and graphene oxide: synthesis, properties, and applications. Advanced Materials, 2010, 22(35): 3906-3924.

[11] YOO E J, KIM J, HOSONO E, et al. Large reversible Li storage of graphene nanosheet families for use in rechargeable lithium on batteries. Nano Letters, 2008, 8(8): 2277-2282.

[12] LOH K P, BAO Q, ANG P K, et al. The chemistry of graphene. Journal of Materials Chemistry, 2010, 12(20): 2277-2289.

[13] MOHANTY N, BERRY V. Graphene-based single-bacterium 
resolution biodevice and DNA transistor: interfacing graphene derivatives with nanoscale and microscale biocomponents. Nano Letters, 2008, 8(12): 4469-4476.

[14] EDA G, CHHOWALLA M. Graphene-based composite thin films for electronics. Nano Letters, 2009, 9(2): 814-818.

[15] MCALLISTER M J, LI J L, ADAMSON D H, et al. Single sheet functionalized graphene by oxidation and thermal expansion of graphite. Chemistry of Materials, 2007, 19(18): 4936-4404.

[16] LI D, MULleR M B, WALlaCE G G, et al. Processable aqueous dispersions of graphene nanosheets. Nature Nanotechnology, 2008, 3(2): 101-105.

[17] MiCHELI D, APOLLO C, PASTORE R, et al. X-band microwave characterization of carbon-based nanocomposite material, absorption capability comparison and RAS design simulation. Composites Science and Technology, 2010, 70(2): 400-409.

[18] LIANG J, WANG Y, HUANG Y, et al. Electromagnetic interference shielding of graphene/epoxy composites. Carbon, 2009, 47(3): 922-925.

[19] YANG L, ZHANG Y C, WANG X, et al. Excellent microwave absorbing property of multiwalled carbon nanotubes with skincore heterostructure formed by outer dominated fluorination. The Journal of Physical Chemistry C, 2018, 122(11): 6357-6367.

[20] NA L, HUANG G W, LI Y Q, et al. Enhanced microwave absorption performance of coated carbon nanotubes by optimizing the $\mathrm{Fe}_{3} \mathrm{O}_{4}$ nanocoating structure. ACS Applied Materials \& Interfaces, 2017, 9(3): 2973-2983.

[21] TIAN X, MENG F B, MENG F C, et al. Synergistic enhancement of microwave absorption using hybridized polyaniline@helical CNTs with dual chirality. ACS Applied Materials \& Interfaces, 2017, 9(18): 15711-15718.

[22] PALLAB B. Graphene and MWCNT: potential candidate for microwave absorbing materials. Journal of Materials Research, 2012, 1(2): 126-132.

[23] WEI L S, KAI L Z, MING J C, et al. A universal permittivityattenuation evaluation diagram for accelerating design of dielectric-based microwave absorption materials: a case of graphene-based composites. Carbon, 2017, 118: 86-97.

[24] DA Y, XING C WEI, YI L X. Tunable microwave absorber based on patterned graphene. IEEE Transactions on Microwave Theory and Techniques, 2017, 65(8): 2819-2826.

[25] BIAN W, HATICE M T, MAJID N, et al. Experimental demonstration of a transparent graphene millimetre wave absorber with $28 \%$ fractional bandwidth at $140 \mathrm{GHz}$. Scientific Reports, 2014, 4(4130): $1-7$.

[26] DAVIDE M, ANTONIO V, ROBERTO P, et al. Synthesis and electromagnetic characterization of frequency selective radar absorbing materials using carbon nano-powders. Carbon, 2014, 77: 756-774.

[27] JING Y F, WANG Y, LI Q H, et al. Enhanced electromagnetic microwave absorption performance of lightweight bowl-like carbon nanoparticles. Industrial \& Engineering Chemistry Research, 2017, 56(40): 11460-11466.

[28] OSMAN B, EMRE O P, NURBEK K, et al. Graphene-enabled electrically switchable radar-absorbing surfaces. Nature Coтmunications, 2015, 6: 6628-1-9.

[29] XIAN J H, ZHI R H, PEI G L, et al. Graphene based tunable fractal Hilbert curve array broadband radar absorbing screen for radar cross section reduction. AIP Advances, 2014, 4: 117103-112.

[30] CHAO W, XI J H, PING X, et al. The electromagnetic property of chemically reduced graphene oxide and its application as microwave absorbing material. Applied Physics Letters, 2011, 98:
072906-1-3.

[31] CHEN C, XI J B, ZHOU E Z, et al. Porous graphene microflowers for high-performance microwave absorption. Nano-Micro Letters, 2018, 10(2): 26-1-11.

[32] HUA L L, YU H G, YUE Z, et al. Achieving tunable electromagnetic absorber via graphene/carbon sphere composites. Carbon, 2016, 110: 130-137.

[33] HUI M L, LIN L, HAI B L, et al. Assembling carbon fibergraphene-carbon fiber hetero-structures into 1D-2D-1D junction fillers and patterned structures for improved microwave absorption. Journal of Physics D: Applied Physics, 2017, 50(13): 135303-1-10.

[34] WEN B, WANG X X, CAO W Q, et al. Reduced graphene oxides: the thinnest and most lightweight materials with highly efficient microwave attenuation performances of the carbon world. Nanoscale, 2014, 6(11): 5754-5761.

[35] CHUN Y C, NEN W P, YI M L, et al. Remarkable microwave absorption performance of graphene at a very low loading ratio. Composites Part B-Engineering, 2017, 114: 395-403.

[36] CHEN C Y, PU N W, LIU Y M, et al. Microwave absorption properties of holey graphene/silicone rubber composites. Composites Part B-Engineering, 2018, 135: 119-128.

[37] WANG Z, LUO J, ZHAO G L. Dielectric and microwave attenuation properties of graphene nanoplatelet-epoxy composites. AIP Advances, 2014, 4(1): 017139-1-9.

[38] VIVEK K S, ANUJ S, MANOJ K P, et al. Microwave absorbing properties of a thermally reduced graphene oxide/nitrile butadiene rubber composite. Carbon, 2012, 50: $2202-2208$.

[39] XIN B, YING H Z, YONG Z. Green approach to prepare graphenebased composites with high microwave absorption capacity. The Journal of Physical Chemistry C, 2011, 115(23): 11673- 11677.

[40] LI J, BI S, SU X J, et al. Preparation and Characterization of Flexibility and Lightweight CF-RGO Composites. 2nd International Conference on New Material and Chemical Industry, 2017, 292: 012093.

[41] KAI C Z, XIN B G, QIAN Z, et al. Pitch carbon coating graphene/ carbon nanotubes lightweight composite and their excellent microwave absorption capacity. Journal of Materials ScienceMaterials in Electronics, 2017, 28(2): 1352-1358.

[42] HONG H C, ZHI Y H, YI H, et al. Synergistically assembled MWCNT/graphene foam with highly efficient microwave absorption in both $\mathrm{C}$ and $\mathrm{X}$ bands. Carbon, 2017, 124: 506-514.

[43] YOU J L, MIAO Y, PIN G Y, et al. Enhanced microwave absorption property of $\mathrm{Fe}$ nanoparticles encapsulated within reduced graphene oxide with different thicknesses. Industrial \& Engineering Chemistry Research, 2017, 56(31): 8872-8879.

[44] JUN F J, FANG L S, KE Z W, et al. Microwave absorbing properties of nickel-coated graphene. Journal of Inorganic Materials, 2011, 26(5): 467-471.

[45] YA R L, SU Y W, DAN L Q, et al. Tunable electromagnetic wave absorption properties of nickel microspheres decorated reduced graphene oxide. Ceramics International, 2017, 43(15): 1290412914.

[46] TING T C, FANG D, JIA Z, et al. Hexagonal and cubic Ni nanocrystals grown on graphene: phase-controlled synthesis, characterization and their enhanced microwave absorption properties. Journal of Materials Chemistry, 2012, 22(30): 1519015197.

[47] YUAN H R, YAN F, LI C Y, et al. Nickel nanoparticle encapsulated in few-layer nitrogen-doped graphene supported by nitrogendoped graphite sheets as a high-performance electromagnetic wave absorbing material. ACS Applied Materials \& Interfaces, 
2018, 10(1): 1399-1407.

[48] GUO H P, JIA Z, SHU L M, et al. Enhancing the electromagnetic performance of Co through the phase-controlled synthesis of hexagonal and cubic Co nanocrystals grown on graphene. ACS Applied Materials \& Interfaces, 2013, 5(23): 12716-12724.

[49] LONG Q, XU Z Q, XIAO H H, et al. A facile synthesis of a cobalt nanoparticle-graphene nanocomposite with high-performance and triple-band electromagnetic wave absorption properties. $R S C$ Advances, 2018, 8(3): 1210-1217.

[50] HUA L L, YU H G, GUANG L W, et al. Interface polarization strategy to solve electromagnetic wave interference issue. ACS Applied Materials \& Interfaces, 2017, 9(6): 5660-5668.

[51] YI D, ZHENG Z, BAO H L, et al. Investigation on the broadband electromagnetic wave absorption properties and mechanism of $\mathrm{Co}_{3} \mathrm{O}_{4}$-nanosheets/reduced-graphene-oxide composite. Nano Research, 2017, 10(3): 980-990.

[52] PAN B L, YING H, LEI W, et al. Hydrothermal synthesis of reduced graphene oxide- $\mathrm{Co}_{3} \mathrm{O}_{4}$ composites and the excellent microwave electromagnetic properties. Material Letters, 2013, 107: 166-169.

[53] BAO P Z, CHUN X L, HAI L, et al. Improving microwave adsorption property of $\mathrm{ZnO}$ particle by doping graphene. Material Letters, 2014, 116: 16-19.

[54] WEI F, YA M W, JUN C C, et al. Reduced graphene oxide decorated with in-situ growing $\mathrm{ZnO}$ nanocrystals: facile synthesis and enhanced microwave absorption properties. Carbon, 2016, 108: $52-60$.

[55] XIAO H L, BIN Q, GUANG B J, et al. Tunable dielectric performance derived from the metal-organic framework/reduced graphene oxide hybrid with broadband absorption. ACS Sustainable Chemistry \& Engineering, 2017, 5(11): 10570-10579.

[56] LONG Z, XIAO H Z, GUANG J Z, et al. Investigation on the optimization, design and microwave absorption properties of reduced graphene oxide/tetrapod-like $\mathrm{ZnO}$ composites. RSC Advances, 2015, 5(14): 10197- 10203.

[57] WEI F, YA M W, JUN C C, et al. Microwave absorbing property optimization of starlike $\mathrm{ZnO} /$ reduced graphene oxide doped by $\mathrm{ZnO}$ nanocrystal composites. Physical Chemistry Chemical Physics, 2017, 19(22): 14596-14605.

[58] MEI K H, XIAO W Y, LUO K, et al. Graphene-wrapped ZnO hollow spheres with enhanced electromagnetic wave absorption properties. Journal of Materials Chemistry A, 2014, 2(39): 16403-16409.

[59] SUKANTA D, SAHU S K, RAMESH O, et al. Reducedgraphene-oxide-and-strontium-titanate-based double-layered composite: an efficient microwave-absorbing material. Bulletin of Materials Science, 2017, 40(2): 301-306.

[60] ZHANG H, TIAN X Y, WANG C P, et al. Facile synthesis of $\mathrm{RGO} / \mathrm{NiO}$ composites and their excellent electromagnetic wave absorption properties. Applied Surface Science, 2014, 314: 228232.

[61] WANG L, XING H L, GAO S T, et al. Porous flower-like $\mathrm{NiO@graphene} \mathrm{composites} \mathrm{with} \mathrm{superior} \mathrm{microwave} \mathrm{absorption}$ properties. Journal of Materials Chemistry C, 2017, 5(8): 20052014.

[62] CHEN C, PAN L M, JIANG S C, et al. Electrical conductivity, dielectric and microwave absorption properties of graphene nanosheets/magnesia composites. Journal of the European Ceramic Society, 2018, 38(4): 1639-1646.

[63] CAN Z, BO C W, JIAN Y X, et al. Microwave absorption properties of $\mathrm{CoS}_{2}$ nanocrystals embedded into reduced graphene oxide. ACS Applied Materials \& Interfaces, 2017, 9(34): 28868-28875.
[64] RUI L Y, BO C W, JIAN Y X, et al. Fabrication of $\mathrm{NiCo}_{2}-$ anchored graphene nanosheets by liquid-phase exfoliation for excellent microwave absorbers. ACS Applied Materials \& Interfaces, 2017, 9(14): 12673-12679.

[65] WANG Y, CHEN D, YIN X, et al. Hybrid of $\mathrm{MoS}_{2}$ and reduced graphene oxide: a lightweight and broadband electromagnetic wave absorber. ACS Applied Materials \& Interfaces, 2015, 7(47): 26226-26231.

[66] PAN B L, YING H, JING Y. et al. Construction of CuS nanoflakes vertically aligned on magnetically decorated graphene and their enhanced microwave absorption properties. ACS Applied Materials \& Interfaces, 2016, 8(8): 5536-5546.

[67] SUKANTA D, GANESH C N, SAHUA S K, et al. Development of $\mathrm{FeCoB} /$ graphene oxide based microwave absorbing materials for X-Band region. Journal of Magnetism and Magnetic Materials, 2015, 384: 224-228.

[68] HE L H, ZHAO Y, XING L Y, et al. Preparation of reduced graphene oxide coated flaky carbonyl iron composites and their excellent microwave absorption properties. RSC Advances, 2018, 8(6): 2971-2977.

[69] ZHU Z T, SUN X, XUE H R, et al. Graphene-carbonyl iron cross-linked composites with excellent electromagnetic wave absorption properties. Journal of Materials Chemistry C, 2014, 2(23): 6582-6591.

[70] ZHANG K, XIE A, SUN M X, et al. Electromagnetic dissipation on the surface of metal organic framework (MOF)/reduced graphene oxide (RGO) hybrids. Materials Chemistry and Physics, 2017, 199: 340-347.

[71] JIANG T Y, QIANG C L, SHI K L, et al. Metal organic framework (MOF)-derived carbonaceous $\mathrm{Co}_{3} \mathrm{O}_{4} / \mathrm{Co}$ microframes anchored on RGO with enhanced electromagnetic wave absorption performances. Synthetic Metals, 2017, 228: 32-40.

[72] QIANG Z, PING C, QI Y, et al. Self-assembly of ternary hollow microspheres with strong wideband microwave absorption and controllable microwave absorption properties. Scientific Reports, 2017, 7: 8388-1-9.

[73] LUO H, CHEN F, WANG F, et al. Preparation and microwave absorption properties of honeycomb core structures coated with composite absorber. AIP Advances, 2018, 8(5): 056635-1-7.

[74] JIA M W, ZHENG M Y, WEN X L, et al. The effect of GO loading on electromagnetic wave absorption properties of $\mathrm{Fe}_{3} \mathrm{O}_{4} /$ reduced graphene oxide hybrids. Ceramics International, 2017, 43(16): 13146-13153.

[75] ERLONG M, JIA J L, NAI Q Z, et al. Preparation of reduced graphene oxide $/ \mathrm{Fe}_{3} \mathrm{O}_{4}$ nanocomposite and its microwave electromagnetic properties. Materials Letters, 2013, 91: 209-212.

[76] LING Y Z, XIAO J Z, XIAO P L, et al. Hydrothermal synthesis of magnetic $\mathrm{Fe}_{3} \mathrm{O}_{4}$ /graphene composites with good electromagnetic microwave absorbing performances. Journal of Magnetism and Magnetic Materials, 2017, 426: 114-120.

[77] GUI Z W, ZHE G, GENG P W, et al. High densities of magnetic nanoparticles supported on graphene fabricated by atomic layer deposition and their use as efficient synergistic microwave absorbers. Nano Research, 2014, 7(5): 704-716.

[78] LIN L, XING M B, ZHI L H, et al. Electromagnetic response of magnetic graphene hybrid fillers and their evolutionary behaviors. Journal of Materials Science: Materials in Electronics, 2016, 27(3): 2760-2772.

[79] WANG X X, MA T, SHU J C, et al. Confinedly tailoring $\mathrm{Fe}_{3} \mathrm{O}_{4}$ clusters-NG to tune electromagnetic parameters and microwave absorption with broadened bandwidth. Chemical Engineering Journal, 2018, 332: 321-330. 
[80] ZHANG K C, GAO X B, ZHANG Q, et al. Carbon coated paramagnetic $\mathrm{Fe}_{3} \mathrm{O}_{4}$ nanoparticles decorated MWCNTs-GNS composites: synthesis, characterization and their excellent electromagnetic absorption properties. Journal of Materials Science: Materials in Electronics, 2018, 29(4): 3401-3410.

[81] XIAN J, BIAO W, YU F W, et al. Facile synthesis of $\mathrm{Fe}_{3} \mathrm{O}_{4} / \mathrm{GCs}$ composites and their enhanced microwave absorption properties. ACS Applied Materials \& Interfaces, 2016, 8(9): 6101-6109.

[82] YING H, XIAO D, SU P L, et al. Magnetic reduced graphene oxide nanocomposite as an effective electromagnetic wave absorber and its absorbing mechanism. Ceramics International, 2016, 42(15): 17116-17122.

[83] LI L Z, XIN X Y, HONG R H, et al. Facile synthesis of iron oxides/ reduced graphene oxide composites: application for electromagnetic wave absorption at high temperature. Scientific Reports, 2015, 5(9298): 1-9.

[84] HAI R C, QIANG Z, PING C, et al. Synthesis and electromagnetic wave absorption properties of matrimony vine-like iron oxide/ reduced graphene oxide prepared by a facile method. Journal of Alloys and Compounds, 2017, 719: 296-307.

[85] LUO K, XIAO W Y, YA J Z, et al. Electromagnetic wave absorption properties of reduced graphene oxide modified by maghemite colloidal nanoparticle clusters. The Journal of Physical Chemistry C, 2013, 117(38): 19701-19711.

[86] QI H, XIAO S Q, HONG B C, et al. Preparation of porous $\mathrm{Fe}_{2} \mathrm{O}_{3}$ nanorods-reduced graphene oxide nanohybrids and their excellent microwave absorption properties. Scientific Reports, 2017, 7: $11213-1-12$.

[87] BIN Q, GUO Y X, DAO R L, et al. Incorporation of dielectric constituents to construct ternary heterojunction structures for high-efficiency electromagnetic response. Journal of Colloid and Interface Science, 2017, 498: 161-169.

[88] ZI Y S, HONG L X, YANG T Z, et al. Synthesis and enhanced microwave-absorbing properties of $\mathrm{SnO}_{2} / \alpha-\mathrm{Fe}_{2} \mathrm{O}_{3} @ \mathrm{RGO}$ composites. Journal of Materials Science: Materials in Electronics, 2017, 28(18): 13896-13904.

[89] DEBABRATA M, SAMYAK D, BARUN K G, et al. Synthesis and microwave absorption properties of $\mathrm{BiFeO}_{3}$ nanowire-rGO nanocomposite and first-principles calculations for insight of electromagnetic properties and electronic structures. The Journal of Physical Chemistry C, 2017, 121(39): 21290-21304.

[90] CHEN W, LIU Q Y, ZHU X X, et al. One-step in situ growth of magnesium ferrite nanorods on graphene and their microwaveabsorbing properties. Applied Organometallic Chemistry, 2018, 32(3): e4017.

[91] XIAO J Z, GUANG S W, WEN Q C, et al. Enhanced microwave absorption property of reduced graphene oxide (RGO)- $\mathrm{MnFe}_{2} \mathrm{O}_{4}$ nanocomposites and polyvinylidene fluoride. ACS Applied Materials \& Interfaces, 2014, 6(10): 7471-7478.

[92] ZEHRA D, ALI D, HUSEYIN K. Synthesis and characterization of structural and magnetic properties of graphene/hard ferrite nanocomposites as microwave-absorbing material. Journal of Materials Science, 2015, 50(3): 1201-1213.

[93] XIAO Y W, MEI L S, HUA L J, et al. Preparation and microwave absorption properties of $\mathrm{CoFe}_{2} \mathrm{O}_{4}$-graphene nanocomposites. Journal of Inorganic Materials, 2014, 8(29): 845-850.

[94] MENG Z, YING H, NA Z, et al. Influence of (RGO)/(ferrite) ratios and graphene reduction degree on microwave absorption properties of graphene composites. Journal of Alloys and Compounds, 2015, 644: 491-501.

[95] XING H L, JUAN F, YA P D, et al. One-pot synthesis of $\mathrm{CoFe}_{2} \mathrm{O}_{4} /$ graphene oxide hybrids and their conversion into
$\mathrm{FeCo} /$ graphene hybrids for lightweight and highly efficient microwave absorber. Journal of Materials Chemistry A, 2015, 3(10): 5535-5546.

[96] PALLAB B, CHAPAL K D. Investigation on microwave absorption capacity of nanocomposites based on metal oxides and graphene. Journal of Materials Science-Materials in Electronics, 2013, 24(6): 1927-1936.

[97] LIN Y, DAI J J, YANG H B, et al. Graphene multilayered sheets assembled by porous $\mathrm{Bi}_{2} \mathrm{Fe}_{4} \mathrm{O}_{9}$ microspheres and the excellent electromagnetic wave absorption properties. Chemical Engineering Journal, 2018, 334: 1740-1748.

[98] MEI B, SU X J, BI S, et al. Study the Effect of $\mathrm{Ni}_{0.5} \mathrm{Zn}_{0.5} \mathrm{Fe}_{2} \mathrm{O}_{4}$ Doped Electromagnetic and Microwave Absorbing Properties on RGO Based Composites. 2018 IOP Conference Series: Materials Science and Engineering, 2018, 292: 012077.

[99] BATEER B, ZHANG J J, ZHANG H C, et al. Easily dispersible $\mathrm{NiFe}_{2} \mathrm{O}_{4} / \mathrm{RGO}$ composite for microwave absorption properties in the X-band. Journal of Electronic Materials, 2018, 47(1): 292-298.

[100] ZHANG Y L, WANG X X, CAO M S. Confinedly implanted $\mathrm{NiFe}_{2} \mathrm{O}_{4}-\mathrm{rGO}$ : cluster tailoring and highly tunable electromagnetic properties for selective-frequency microwave absorption. Nano Research, 2018, 11(3): 1426-1436.

[101] YAN F, GUO D, ZHANG S, et al. An ultra-small $\mathrm{NiFe}_{2} \mathrm{O}_{4}$ hollow particle/graphene hybrid: fabrication and electromagnetic wave absorption property. Nanoscale, 2018, 10(6): 2697-2703.

[102] KAI C Z, XIN B G, QIAN Z, et al. Synthesis, characterization and electromagnetic wave absorption properties of asphalt carbon coated graphene/magnetic $\mathrm{NiFe}_{2} \mathrm{O}_{4}$ modified multi-wall carbon nanotube composites. Journal of Alloys and Compounds, 2017, 721: 268-275.

[103] BATRAKOV K, KUZHIR P, MAKSIMENKO S, et al. Flexible transparent graphene/polymer multilayers for efficient electromagnetic field absorption. Scientific Reports, 2014, 4: 7191-1-5.

[104] MANUELA L, ROLAND Y T, SIU H T, et al. Configurable three-dimensional boron nitride-carbon architecture and its tunable electronic behavior with stable thermal performances. Small, 2014, 10(15): 2992-2999.

[105] BO Z G, WEI L, YUAN L Y, et al. Enhanced microwave absorption properties of graphite nanoflakes by coating hexagonal boron nitride nanocrystals. Applied Surface Science, 2017, 420: 858867.

[106] QUAN L, QIN F X, ESTEVEZ D, et al. Magnetic graphene for microwave absorbing application: towards the lightest graphenebased absorber. Carbon, 2017, 125: 630-639.

[107] LUO H, ZENG S F, TAN Y Q, et al. Mechanism of microwave dielectric response in carbon nanofibers enabled BCN composites. Journal of Materials Science: Materials in Electronics, 2016, 27(10): 10435-10441.

[108] YUE K, ZHEN H J, TIAN M, et al. Hybrids of reduced graphene oxide and hexagonal boron nitride: lightweight absorbers with tunable and highly efficient microwave attenuation properties. ACS Applied Materials \& Interfaces, 2016, 8(47): 32468-32476.

[109] YUE K, ZENG Y C, DONG J Z, et al. Incorporate boron and nitrogen into graphene to make $\mathrm{BCN}$ hybrid nanosheets with enhanced microwave absorbing properties. Carbon, 2013, 61: 200208.

[110] YAN W, HONG Y Z, YAN B C, et al. Design of hollow $\mathrm{ZnFe}_{2} \mathrm{O}_{4}$ microspheres@graphene decorated with $\mathrm{TiO}_{2}$ nanosheets as a high-performance low frequency absorber. Materials Chemistry and Physics, 2017, 202: 184-189.

[111] YA R W, YONG P P, YU S, et al. Excellent microwave absorption property of the $\mathrm{CoFe}_{2} \mathrm{O}_{4} / \mathrm{Y}_{3} \mathrm{Fe}_{5} \mathrm{O}_{12}$ ferrites based on graphene. 
Journal of Materials Science: Materials in Electronics, 2017, 28(17): $12866-12872$.

[112] HAI B Y, TING Y, YING L, et al. Microwave absorbing properties of the ferrite composites based on graphene. Journal of Alloys and Compounds, 2016, 683: 567-574.

[113] PAN B L, YING H, XU S. Excellent electromagnetic absorption properties of Poly(3,4-ethylenedioxythiophene)-reduced graphene oxide- $\mathrm{CO}_{3} \mathrm{O}_{4}$ composites prepared by a hydrothermal method. ACS Applied Materials \& Interfaces, 2013, 5(23): 12355-12360.

[114] HAI B Y, JING J D, XIAO L, et al. Synthesis and enhanced microwave absorption properties of $\mathrm{PVB} / \mathrm{Co}_{2} \mathrm{Z} / \mathrm{RGO}$ layered composite. Journal of Alloys and Compounds, 2017, 714: 537-545.

[115] QING Y C, MIN D D, ZHOU Y Y, et al. Graphene nanosheet- and flake carbonyl iron particle-filled epoxy-silicone composites as thin-thickness and wide-bandwidth microwave absorber. Carbon, 2015, 86: 98-107.

[116] JIN S L, WEI B L, JONGHWAN S, et al. Superb electromagnetic wave absorbing composites based on large-scale graphene and carbon nanotube films. Scientific Reports, 2017, 7: 2349-1-10.

[117] XIE H, YAN X, XIA L, et al. A three-dimensional graphene/ $\mathrm{Fe}_{3} \mathrm{O}_{4} /$ carbon microtube of sandwich-type architecture with improved wave absorbing performance. Scripta Materialia, 2016, 120: $107-111$.

[118] PALLAB B, SAPTARSHI D, MRINAL K K, et al. Graphene and MWCNT based bi-functional polymer nanocomposites with enhanced microwave absorption and supercapacitor property. Materials Research Bulletin, 2015, 66: 200-212.

[119] QING Q, YU M H, MING Z X, et al. Synthesis and microwave absorption properties of sandwich-type $\mathrm{CNTs} / \mathrm{Fe}_{3} \mathrm{O}_{4} / \mathrm{RGO}$ composite with $\mathrm{Fe}_{3} \mathrm{O}_{4}$ as a bridge. Journal of Materials Science: $\mathrm{Ma}$ terials in Electronics, 2017, 28(20): 15043-15049.

[120] HUI Z, MIAO H, PING C, et al. 3D and ternary rGO/MCNTs/ $\mathrm{Fe}_{3} \mathrm{O}_{4}$ composite hydrogels: synthesis, characterization and their electromagnetic wave absorption properties. Journal of Alloys and Compounds, 2016, 665: 381-387.

[121] DAN P S, QUAN Z, YAN P W, et al. Controllable synthesis of porous $\mathrm{Fe}_{3} \mathrm{O}_{4} @ \mathrm{ZnO}$ sphere decorated graphene for extraordinary electromagnetic wave absorption. Nanoscale, 2014, 6(12): $6557-$ 6562 .

[122] MIAO L, XIAN J C, SHUI R Z, et al. Ternary composites $\mathrm{RGO} / \mathrm{MoS}_{2} @ \mathrm{Fe}_{3} \mathrm{O}_{4}$ : synthesis and enhanced electromagnetic wave absorbing performance. Journal of Materials Science: Materials in Electronics, 2017, 28(22): 16802-16812.

[123] KAI C Z, XIN B G, QIAN Z, et al. Preparation and microwave absorption properties of asphalt carbon coated reduced graphene oxide/magnetic $\mathrm{CoFe}_{2} \mathrm{O}_{4}$ hollow particles modified multi-wall carbon nanotube composites. Journal of Alloys and Compounds, 2017, 723: 912-921.

[124] XIAO S Q, QI H, HONG B C, et al. Hetero-nanostructured Co@carbon nanotubes-graphene ternary hybrids: synthesis, electromagnetic and excellent microwave absorption properties. Scientific Reports, 2016, 6: 37972-1-15.

[125] REN F, ZHU G M, WU G L, et al. Effects of surfactant treatment on mechanical and microwave absorbing properties of graphene nanosheets multiwalled carbon nanotubes cyanate ester composites. Polymer Composites, 2018, 39(1): 110-118.

[126] JIA S H, YAN X L, XIAO Y L, et al. Graphene/MWNT/ poly(p-phenylenebenzobisoxazole) multiphase nanocomposite via solution prepolymerization with superior microwave absorption properties and thermal stability. Journal of Physical Chemistry C, 2017, 121(2): 1072-1081.

[127] XIAO D W, BING Z L, YANG Z, et al. Synthesis of flake shaped carbonyl iron/reduced graphene oxide/polyvinyl pyrrolidone ternary nanocomposites and their microwave absorbing properties. Journal of Alloys and Compounds, 2017, 695: 508-519.

[128] XIAO D W, XU L L, BING Z L, et al. One-pot preparation of reduced graphene oxide/carbonyl iron/polyvinyl pyrrolidone ternary nanocomposite and its synergistic microwave absorbing properties. Materials Letters, 2017, 188: 280-283.

[129] CHIH C C, WEN F L, YU H N, et al. Microwave absorbing properties of flake-shaped carbonyl iron/reduced graphene oxide/ epoxy composites. Materials Research Bulletin, 2017, 96(SI): $81-85$.

[130] XIANG N C, FAN C M, ZUO W Z, et al. One-step synthesis of graphene/polyaniline hybrids by in situ intercalation polymerization and their electromagnetic properties. Nanoscale, 2014, 6(14): 8140-8148.

[131] YI K Z, WEI Z, ZI H P, et al. Graphene-doped polyaniline nanocomposites as electromagnetic wave absorbing materials. Journal of Materials Science: Materials in Electronics, 2017, 28(15): 10921-10928.

[132] YU H L, WANG T S, WEN B, et al. Graphene/polyaniline nanorod arrays: synthesis and excellent electromagnetic absorption properties. Journal of Materials Chemistry, 2012, 22(40): 21679 21685.

[133] PING Y, JIE M, JUAN C, et al. Facile synthesis and excellent electromagnetic wave absorption properties of flower-like porous $\mathrm{RGO} / \mathrm{PANI} / \mathrm{Cu}_{2} \mathrm{O}$ nanocomposites. Journal of Materials Science, 2017, 52(22): 13078-13090.

[134] PAN B L, YING H, LEI W, et al. Preparation and excellent microwave absorption property of three component nanocomposites: polyaniline-reduced graphene oxide- $\mathrm{Co}_{3} \mathrm{O}_{4}$ nanoparticles. Synthetic Metals, 2013, 177: 89-93.

[135] PAN B L, YING H, XIANG Z, et al. Synthesis, characterization and excellent electromagnetic wave absorption properties of graphene@ $\mathrm{CoFe}_{2} \mathrm{O}_{4} @$ polyaniline nanocomposites. Synthetic Metals, 2015, 201: 76-81.

[136] XING L C, SHU H Q. Preparation and microwave absorbing properties of polyaniline $/ \mathrm{NiFe}_{2} \mathrm{O}_{4} /$ graphite nanosheet composites via Sol-Gel reaction and in situ polymerization. Journal of Sol-Gel Science and Technology, 2017, 81(3): 824-830.

[137] PALLAB B, SAPTARSHI D, GOUTAM H, et al. Graphene decorated with hexagonal shaped M-type ferrite and polyaniline wrapper: a potential candidate for electromagnetic wave absorbing and energy storage device applications. RSC Advances, 2014, 4(33): 17039-17053.

[138] LI J S, DUAN Y, LU W B, et al. Polyaniline-stabilized electromagnetic wave absorption composites of reduced graphene oxide on magnetic carbon nanotube film. Nanotechnology, 2018, 29(15): 155201-1-9.

[139] LEI W, YING H, CHAO L, et al. Hierarchical composites of polyaniline nanorod arrays covalently-grafted on the surfaces of graphene@ $\mathrm{Fe}_{3} \mathrm{O}_{4} @ \mathrm{C}$ with high microwave absorption performance. Composites Science and Technology, 2015, 108: 1-8.

[140] JUAN L, LEI W, DONG Z, et al. Reduced graphene oxide modified mesoporous $\mathrm{FeNi}$ alloy/carbon microspheres for enhanced broadband electromagnetic wave absorbers. Materials Chemistry Frontiers, 2017, 1(9): 1786-1794.

[141] SU H Z, YING H, JING Y, et al. Fabrication of ternary CoNi@SiO $@$ RGO composites with enhanced electromagnetic (EM) wave absorption performances. Journal of Materials Science: Materials in Electronics, 2017, 28(24): 18558-18567.

[142] BIN Q, CHUN L Z, CHUN Y L, et al. Coupling hollow $\mathrm{Fe}_{3} \mathrm{O}_{4}-\mathrm{Fe}$ nanoparticles with graphene sheets for high-performance elec- 
tromagnetic wave absorbing material. ACS Applied Materials \& Interfaces, 2016, 8(6): 3730-3735.

[143] YU L R, CHUN L Z, SHEN Z, et al. Three-dimensional $\mathrm{SiO}_{2} @ \mathrm{Fe}_{3} \mathrm{O}_{4}$ core/shell nanorod array/graphene architecture: synthesis and electromagnetic absorption properties. Nanoscale, 2013, 5(24): 12296-12303.

[144] SU P L, YING H, XIAO D, et al. Synthesis of core-shell $\mathrm{FeCo@SiO}$. particles coated with the reduced graphene oxide as an efficient broadband electromagnetic wave absorber. Journal of Materials Science: Materials in Electronics, 2017, 28(21): 15782-15789.

[145] JIAN T F, YAN H H, YE C W, et al. Synthesis of hierarchical $\mathrm{ZnFe}_{2} \mathrm{O}_{4} @ \mathrm{SiO}_{2} @ \mathrm{RGO}$ core-shell microspheres for enhanced electromagnetic wave absorption. ACS Applied Materials \& Interfaces, 2017, 9(16): 14103-14111.

[146] BIN Z, JUN W, XIAO G S, et al. Enhanced microwave absorption properties of epoxy composites containing graphene decorated with core-shell $\mathrm{Fe}_{3} \mathrm{O}_{4} @$ polypyrrole nanoparticles. Journal of Materials Science: Materials in Electronics, 2017, 28(16): 12122-12131.

[147] PAN B L, YING H, XIANG Z. Synthesis of graphene@ branchinglike polypyrrole@ $\mathrm{CoFe}_{2} \mathrm{O}_{4}$ composites and their excellent electromagnetic wave absorption properties. Materials Letters, 2014, 36: 298-301.

[148] BIN L, JIN H L, LU F W, et al. Ultralight graphene aerogel enhanced with transformed micro-structure led by polypyrrole nano-rods and its improved microwave absorption properties. Composites Part A: Applied Science and Manufacturing, 2017, 97: 141-150.

[149] HUANG Y, YAN J, ZHOU S H, et al. Preparation and electromagnetic wave absorption properties of $\mathrm{CoNi} @ \mathrm{SiO}_{2}$ microspheres decorated grapheme-polyaniline nanosheets. Journal of Materials Science: Materials in Electronics, 2018, 29(1): 70-79.

[150] LEI W, JIAN F Z, HAI B Y, et al. Fabrication of hierarchical graphene@ $\mathrm{Fe}_{3} \mathrm{O}_{4} @ \mathrm{SiO}_{2} @$ polyaniline quaternary composite and its improved electrochemical performance. Journal of Alloys and Compounds, 2015, 634: 232-238.

[151] LEI W, YING H, XU S, et al. Synthesis and microwave absorption enhancement of graphene@ $\mathrm{Fe}_{3} \mathrm{O}_{4} @ \mathrm{SiO}_{2} @ \mathrm{NiO}$ nanosheets hierarchical structures. Nanoscale, 2014, 6(6): 3157-3164.

[152] YU L R, HONG Y W, MING M L, et al. Quaternary nanocomposites consisting of graphene, $\mathrm{Fe}_{3} \mathrm{O}_{4} @ \mathrm{Fe}$ core@shell, and $\mathrm{ZnO}$ nanoparticles: synthesis and excellent electromagnetic absorption properties. ACS Applied Materials \& Interfaces, 2012, 4(12): 6436-6442.

[153] YAN W, XIN M W, WEN Z Z, et al. Fabrication and enhanced electromagnetic wave absorption properties of sandwich-like graphene@NiO@PANI decorated with Ag particles. Synthetic Metals, 2017, 229: 82-88.

[154] YAN W, XIN M W, WEN Z Z, et al. 3D heterostructure of graphene@ $\mathrm{Fe}_{3} \mathrm{O}_{4} @ \mathrm{WO}_{3} @ \mathrm{PANI}$ : preparation and excellent microwave absorption performance. Synthetic Metals, 2017, 231: $7-14$.

[155] TING K Z, XIANG L J, WEN B J, et al. Synthesis and electromagnetic wave absorption property of amorphous carbon nanotube networks on a $3 \mathrm{D}$ graphene aerogel/ $\mathrm{BaFe}_{12} \mathrm{O}_{19}$ nanocomposite. Journal of Alloys and Compounds, 2017, 708: 115-122.

[156] CHAO L, YING H, JUN J C. Dopamine-assisted one-pot synthesis of graphene@Ni@C composites and their enhanced microwave absorption performance. Materials Letters, 2015, 154: 136-139.

[157] CHUN L Z, SHEN Z, YUE S, et al. Incorporation of CoO@Co yolk-shell nanoparticles and $\mathrm{ZnO}$ nanoparticles with graphene sheets as lightweight and high performance electromagnetic wave absorbing material. Journal of Alloys and Compounds, 2017, 711: 552-559.

[158] YI Z, YI H, TENG F Z, et al. Broadband and tunable high-performance microwave absorption of an ultralight and highly compressible graphene foam. Advanced Materials, 2015, 27(12): 2049-2053.

[159] YI Z, YI H, HONG H C, et al. Composition and structure control of ultralight graphene foam for high-performance microwave absorption. Carbon, 2016, 105: 438-447.

[160] WEI L S, XIAO T G, LI Z F, et al. Strong and thermostable polymeric graphene/silica textile for lightweight practical microwave absorption composites. Carbon, 2016, 100: 109-117.

[161] CHUN Y C, AI B Z, LI C D, et al. A three-dimensional absorber hybrid with polar oxygen functional groups of MWNTs/graphene with enhanced microwave absorbing properties. Composites Part B: Engineering, 2017, 108: 386-392.

[162] WANG L, HUANG Y, LI C, et al. A facile one-pot method to synthesize a three-dimensional graphene@carbon nanotube composite as a high-efficiency microwave absorber. Physical Chemistry Chemical Physics, 2015, 17(3): 2228-2234.

[163] WANG C H, DING Y J, YUAN Y, et al. Graphene aerogel composites derived from recycled cigarette filters for electromagnetic wave absorption. Journal of Materials Chemistry C, 2015, 3(45): 11893-11901.

[164] LIU W W, LI H, ZENG Q P, et al. Fabrication of ultralight three-dimensional graphene networks with strong electromagnetic wave absorption properties. Journal of Materials Chemistry A, 2015, 3(7): 3739-3747.

[165] XIE A, WU F, SUN M X, et al. Self-assembled ultralight threedimensional polypyrrole aerogel for effective electromagnetic absorption. Applied Physics Letters, 2015, 106(22): 222902-1-5.

[166] WANG Y, WU X M, ZHANG W Z. Synthesis and highperformance microwave absorption of graphene foam/polyaniline nano-rods. Materials Letters, 2016, 165: 71-74.

[167] HAN M K, YIN X W, DUAN W Y, et al. Hierarchical graphene/ $\mathrm{SiC}$ nanowire networks in polymer-derived ceramics with enhanced electromagnetic wave absorbing capability. Journal of the European Ceramic Society, 2016, 36: 2695-2703.

[168] ZHANG N, HUANG Y, WANG M Y. 3D ferromagnetic graphene nanocomposites with $\mathrm{ZnO}$ nanorods and $\mathrm{Fe}_{3} \mathrm{O}_{4}$ nanoparticles co-decorated for efficient electromagnetic wave absorption. Composites Part B: Engineering, 2018, 136: 135-142.

[169] LI J S, XIE Y Z, LU W B, et al. Flexible electromagnetic wave absorbing composite based on $3 \mathrm{D}$ rGO-CNT- $-\mathrm{Fe}_{3} \mathrm{O}_{4}$ ternary films. Carbon, 2018, 129: 76-84.

[170] ZENG Q, XU D W, CHEN P, et al. 3D graphene-Ni microspheres with excellent microwave absorption and corrosion resistance properties. Journal of Materials Science: Materials in Electronics, 2018, 29(3): 2421-2433.

[171] CHANG Q S, XIAO W Y, MEI K H, et al. Three-dimensional reduced graphene oxide foam modified with $\mathrm{ZnO}$ nanowires for enhanced microwave absorption properties. Carbon, 2017, 116: 50-58.

[172] MEI K H, XIAO W Y, ZE X H, et al. Flexible and thermostable graphene/SiC nanowire foam composites with tunable electromagnetic wave absorption properties. ACS Applied Materials \& Interfaces, 2017, 9(13): 11803-11810.

[173] SHUN D, JUN T S, XING H Z, et al. Strong contribution of in situ grown nanowires to enhance the thermos-stabilities and microwave absorption properties of porous graphene foams under 
different atmospheres. Journal of Materials Chemistry C, 2017, 5(45): 11837-11846.

[174] JIAN N M, WEI L, BIN Q, et al. Incorporation of the polarization point on the graphene aerogel to achieve strong dielectric loss behavior. Journal of Colloid and Interface Science, 2017, 504: 479-484.

[175] YU T L, JUN L X, HONG L, et al. Solvothermal synthesis, electromagnetic and electrochemical properties of jellylike cylinder graphene- $\mathrm{Mn}_{3} \mathrm{O}_{4}$ composite with highly coupled effect. Journal of Solid State Chemistry, 2017, 256: 256-265.

[176] HU C G, MOU Z Y, LU G W, et al. 3D graphene- $\mathrm{Fe}_{3} \mathrm{O}_{4}$ nanocomposites with high-performance microwave absorption. Physical Chemistry Chemical Physics, 2013, 15(31): 13038-13043.

[177] XING M L, ZHAO J Y, RYO I, et al. Single-source-precursor synthesis and electromagnetic properties of novel RGO-SiCN ceramic nanocomposites. Journal of Materials Chemistry C, 2017, 5(31): 7950-7960.

[178] QING Y C, WEN Q L, LUO F, et al. Temperature dependence of the electromagnetic properties of graphene nanosheet reinforced alumina ceramics in the X-band. Journal of Materials Chemistry C, 2016, 4(22): 4853-4862.

[179] KONG L, YIN X W, YUAN X Y, et al. Electromagnetic wave absorption properties of graphene modified with carbon nanotube/ poly(dimethyl siloxane) composites. Carbon, 2014, 73: 185-193.

[180] REN F, ZHU G M, WU G L, et al. Effects of surfactant treatment on mechanical and microwave absorbing properties of graphene nanosheets/multiwalled carbon nanotubes/cyanate ester composites. Polymer Composites, 2018, 39(1): 110-118.

[181] LV X J, DUAN Y P, CHEN G Q. Electromagnetic wave absorption properties of cement-based composites filled with graphene nano-platelets and hollow glass microspheres. Construction and
Building Materials, 2018, 162: 280-285.

[182] QING Y C, NAN H Y, MA L Y, et al. Double-layer structure combined with FSS design for the improvement of microwave absorption of $\mathrm{BaTiO}_{3}$ particles and graphene nanoplatelets filled epoxy coating. Journal of Alloys and Compounds, 2018, 739: $47-51$.

[183] YUAN H R, YAN F, LI C Y, et al. Nickel nanoparticle encapsulated in few-layer nitrogen-doped graphene supported by nitrogendoped graphite sheets as a high-performance electromagnetic wave absorbing material. ACS Applied Materials \& Interfaces, 2018, 10(1): 1399-1407.

[184] MA D Y, LI X X, GUO Y X, et al. Preparation and microwaveinfrared absorption of reduced graphene oxide/Cu-Ni ferrite/ $\mathrm{Al}_{2} \mathrm{O}_{3}$ composites. Materials Research Express, 2018, 5(1): 016106-1-7.

[185] FU C, HE D W, WANG Y S, et al. Synthesis and microwave absorption properties of graphene-oxide(GO)/polyaniline nanocomposite with gold nanoparticles. Chinese Physics B, 2015, 24(8): 087801-1-5.

[186] REZA M, SOHRAB R, MIR S S D, et al. Microwave absorption properties of $\mathrm{GO}$ nanosheets- $x \mathrm{CoFe}_{2} \mathrm{O}_{4}-\mathrm{NiO}$ nanocomposites based on epoxy resin: optimization using Taguchi methodology. Journal of Materials Science: Materials in Electronics, 2018, 29(6): 4583-4595.

[187] ZHANG W D, ZHANG X, QIAO Y L, et al. Covalently bonded GNPs-NH-PANI nanorod arrays modified by $\mathrm{Fe}_{3} \mathrm{O}_{4}$ nanoparticles as high-performance electromagnetic wave absorption materials. Materials Letters, 2018, 216: 101-105.

[188] Huang Z Y, Chen H H, Huang Y, et al. Ultra-broadband wide-angle terahertz absorption properties of 3D graphene foam. Advanced Functional Materials, 2018, 28(2): 1704363-1-8. 